\title{
Analysis of Shell Structures under Transient Loading using Adaptivity in Time and Space
}

\author{
J. Neumann, K. Schweizerhof \\ Universität Karlsruhe, Institut für Mechanik
}

1999

Institut für Mechanik

Kaiserstr. 12, Geb. 20.30

76128 Karlsruhe

Tel.: +49 (0) 721/ 608-2071

Fax: +49 (0) 721/ 608-7990

E-Mail: ifm@uni-karlsruhe.de

www.ifm.uni-karlsruhe.de 


\title{
Analysis of Shell Structures under Transient Loading using Adaptivity in Time and Space
}

\author{
Jens Neumann and Karl Schweizerhof \\ Institute for Mechanics \\ University Karlsruhe, Germany \\ e-mail: Karl.Schweizerhof@bau-verm.uni-karlsruhe.de
}

Key words: Transient analysis, solid mechanics, error estimation, adaptive mesh modification, FE-analysis.

\begin{abstract}
The adaptive analysis of structures under transient loading leads to the question, which time integration scheme, Finite Elements or Finite Differences, is most favorably combined with an adaptive spatial FE discretization. In order to judge this, the properties of different Discontinuous Galerkin and the standard Newmark method are investigated first, also concerning efficency. In particular, the damping and dispersion effects are discussed in detail for various types of problems. It must be noted that the type of problem has to be carefully checked in order to apply the most appropriate and efficient time integration scheme. It is shown that e.g. for the wave propagation problems the Discontinuous Galerkin method with linear approximations (DG P1-P1) has to be favored when adaptivity in space is applied. Finally, an adaptive time step modification scheme is presented and applied to various problems.
\end{abstract}




\section{Introduction}

Complex solid mechanics problems with external time dependent loading and other excitation often require a very high solution effort, and numerical schemes have to be used for spatial discretization and time integration. As the loading of the different parts of the structures varies not only within the structure but also with time, adaptive solution procedures seem to be very attractive to achieve a high efficiency of the solution process with controlled errors.

The standard schemes for error estimation in FE meshes known from statics cannot be applied without considering the kinetic and damping effects as well as the time integration effects. It is particular important to keep in mind that with kinetic effects also many physical phenomena appear which have to be well represented by the solution methods.

The contribution is therefore directed towards the capturing of kinetic effects, respectively the interaction of the numerical solution schemes with various details of the solution. First, Discontinuous Galerkin time integration methods are compared with standard Newmark type methods for different types of problems using stationary and adaptively modified meshes. Then, the focus is on dispersion effects, in particular introduced by the spatial discretization and their representation with the two integration methods discussed before. This is followed by an investigation, if and how mesh adaptation changes with the effects observed with stationary meshes. Finally, a simple and efficient time step modification method is presented and discussed using a numerical example.

\section{Time integration - Discontinuous Galerkin vs. Newmark type methods}

In order to take also kinetic aspects into account Cauchy's first equation of motion for solids has to be used to describe the behavior of solid type structures under general loading:

$$
\operatorname{div} \boldsymbol{\sigma}+\rho \mathbf{d}=\rho \ddot{\mathbf{u}},
$$

with : $\quad \boldsymbol{\sigma}$ : linear Cauchy-stress tensor,

d : acceleration field (body force),

$\left.\mathbf{u}(x, 0)=\mathbf{u}_{0}, \quad\right\}$ initial displacements, $\left.\dot{\mathbf{u}}(x, 0)=\dot{\mathbf{u}}_{0}, \quad\right\}$ initial velocities

and : $\left.\begin{array}{l}\mathbf{u}(x, t)=\mathbf{u}_{1}(t) ; \forall \mathbf{x} \in \Gamma_{1}, \\ \boldsymbol{\sigma}(x, t) \mathbf{n}=\mathbf{p}(t) ; \forall \mathbf{x} \in \Gamma_{2} .\end{array}\right\}$ boundary conditions

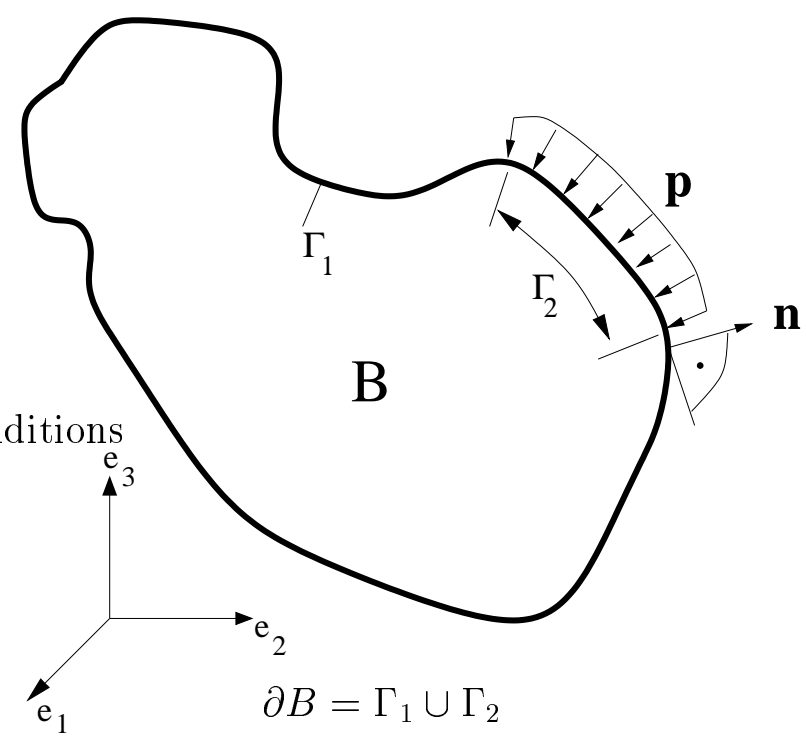

Figure 1: Solid under general loading. thus the discretization in time and space is performed separately, see e.g. Hughes [8], 
Zienkiewicz/Taylor [27], e.g. both with FE method, see Hulbert/Hughes [9]. Other methods, though promising, see Hulbert [12], have not yet been successful in practice.

For the spatial discretization the standard Bubnov-Galerkin formulation is chosen:

$$
\int_{B} \mathbf{v}(\operatorname{div} \boldsymbol{\sigma}+\rho \mathbf{d}-\rho \ddot{\mathbf{u}}) d V=0 .
$$

Applying the Green-Gauss theorem for the first term of (2) :

$$
\int_{B} \mathbf{v} d i v \boldsymbol{\sigma} d V=\int_{\Gamma_{2}} \mathbf{v} \boldsymbol{\sigma} \mathbf{n} d A-\int_{B} \nabla \mathbf{v} \boldsymbol{\sigma} d V,
$$

leads to the well known weak form of eq. (1). The following investigations are restricted to linear behavior with the linear strain tensor $\boldsymbol{\epsilon}: \boldsymbol{\epsilon}=1 / 2\left(\nabla \mathbf{u}+\nabla \mathbf{u}^{T}\right)$ and linear material laws, e.g. the St.-Venant Kirchhoff model $\boldsymbol{\sigma}=\mathbb{C}: \boldsymbol{\epsilon}$. The test space $Y$ and the space of the trial functions $X$ are defined as: $\mathbf{v} \in Y ; Y=\left\{\left(H^{1}\right)^{n}, \mathbf{v}=0\right.$ on $\left.\Gamma_{1}\right\}, \quad \mathbf{u} \in X ; X=$ $\left\{\left(H^{1}\right)^{n}, \mathbf{u}(x)=\mathbf{u}_{1}\right.$ on $\left.\Gamma_{1}\right\}$.

Thus, the first step of semidiscretization leads to a second order ordinary differential equation system (ODE), with the assumption of modal damping $\mathbf{D}=a \mathbf{M}+b \mathbf{K}$ we obtain:

$$
\mathbf{M} \ddot{\mathbf{u}}(t)+\mathbf{D} \dot{\mathbf{u}}(t)+\mathbf{K u}(t)=\mathbf{f}(t) .
$$

In general this ODE system (4) has to be integrated numerically.

\section{$3 \quad$ Numerical time integration}

Mainly either Finite Differences (FD) or Finite Elements in Time (FET) are used for the time integration, see [17], Chung [3], Hilber [6], Hoff [7], Hughes [9], Hulbert [11], Zienkiewicz [26]. Both methods have in common that the total integration interval $t \in[0, T]$ is divided into 'local' intervals $I_{n}=\left[t_{n}, t_{n+1}\right]$ with a time step size $\Delta t=t_{n+1}-t_{n}$. Thus a piecewise integration is performed. In the following, the major differences of both approaches are investigated, in particular with respect to spatial adaptivity.

The most popular FD scheme is the basic approach of Newmark [19]:

$$
\begin{aligned}
& \mathbf{u}_{n+1}=\mathbf{u}_{n}+\Delta t \dot{\mathbf{u}}_{n}+\Delta t^{2}\left((0.5-\beta) \ddot{\mathbf{u}}_{n}+\beta \mathbf{u}_{n+1}\right), \\
& \dot{\mathbf{u}}_{n+1}=\dot{\mathbf{u}}_{n}+\Delta t\left((1-\gamma) \ddot{\mathbf{u}}_{n}+\gamma \dot{\mathbf{u}}_{n+1}\right) .
\end{aligned}
$$

The parameters $\beta$ and $\gamma$ control the accuracy, artificial damping and the stability of the method.

Standard FET have been proposed already since long e.g. by Oden [21], who used the Hamiltonian and the variation as the starting point of his derivations. Zienkiewicz [26] showed in a paper the equivalence between the Newmark scheme and a distinct FET method. All these schemes suggested were based on continuity assumptions at the beginning resp. end of the local time interval. More recent developments, see Hughes/ 
Hulbert [9], Johnson [13] and Aharoni/Bar-Yoseph [1], as well as Wiberg/Li [16] and Ruge [23] allow discontinuities of the state variables at the internal boundaries with the penalty of an enlargement of the equation systems. In general, various ways to develop the final algebraic system of equations can be chosen. If the ODE (4) is directly taken as starting point, a so-called 2-field formulation with displacements and velocities as variables seems to have some advantages:

$$
\begin{aligned}
\dot{\mathbf{u}} & =\mathbf{v}, \\
\mathbf{M} \dot{\mathbf{v}}+\mathbf{D v}+\mathbf{K u} & =\mathbf{f} .
\end{aligned}
$$

A Petrov-Galerkin method is used for the solution of this coupled system; both equations are weighted with different test functions $\mathbf{w}_{u}$ and $\mathbf{w}_{v}$ and then integration is performed over the time interval $\Delta t$. In addition, the jumps in displacements $\Delta \mathbf{u}_{n}^{+}-\mathbf{u}_{n}^{-}$and velocities $\Delta \mathbf{v}_{n}^{+}-\mathbf{v}_{n}^{-}$at the beginning of the time interval are introduced, see figure 2 , and the following (variational) form is obtained:

$$
\begin{gathered}
\int_{\tau} \mathbf{w}_{v}(\mathbf{M} \dot{\mathbf{v}}+\mathbf{D} \mathbf{v}+\mathbf{K u}) d \tau+\int_{\tau} \mathbf{w}_{u} \mathbf{K}(\dot{\mathbf{u}}-\mathbf{v}) d \tau+\mathbf{w}_{v}^{+n} \cdot \mathbf{M} \mathbf{v}_{n}^{+n}+\mathbf{w}_{u}^{+n} \cdot \mathbf{K} \mathbf{u}_{n}^{+n}= \\
\int_{\tau} \mathbf{w}_{v} \mathbf{f}(t) d \tau+\mathbf{w}_{v}^{+n} \cdot \mathbf{M} \mathbf{v}_{n}^{-n}+\mathbf{w}_{u}^{+n} \cdot \mathbf{K} \mathbf{u}_{n}^{-n}, \quad \forall\left\{\mathbf{w}_{u}, \mathbf{w}_{v}\right\} \in\left\{H^{1}(0, \Delta t) \times H^{1}(0, \Delta t)\right\} .
\end{gathered}
$$

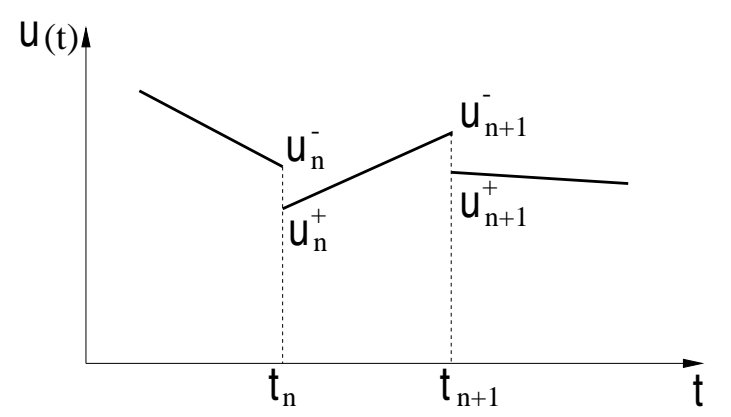

Figure 2: Time discretization involving jumps in displacements.

Due to the jump terms other test functions with less restrictions can be used, as continuity is not required. Such schemes are known as Discontinuous-Galerkin (DG) methods, see Johnson/Pitkäranta [14]. The test and trial functions for displacements $\mathcal{S}_{u}$ and velocities $\mathcal{S}_{v}$ can be chosen as any order polynomials. Usually, low order schemes -linear or quadratic- are preferred $[11,16,23]$ due to several aspects discussed in the following. E.g. if linear functions are chosen for both displacements and velocities, the so-called $D G$ $P 1-P 1$ method is obtained:

$\mathcal{S}_{u}=\left\{\mathbf{u}_{h}, \mathbf{w}_{u} \in \bigcup_{n=1}^{N}\left(\mathcal{P}^{1}\left(I_{n}\right)\right)^{n e q}\right\}, \mathcal{S}_{v}=\left\{\mathbf{v}_{h}, \mathbf{w}_{v} \in \bigcup_{n=1}^{N}\left(\mathcal{P}^{1}\left(I_{n}\right)\right)^{n e q}\right\}$

$\mathcal{P}^{1}$ is the space of first order polynomials.

$$
\begin{aligned}
& \mathbf{u}_{h}=(1-\tau) \mathbf{u}_{n}^{+}+\tau \mathbf{u}_{n+1}^{-}, \quad \mathbf{v}_{h}=(1-\tau) \mathbf{v}_{n}^{+}+\tau \mathbf{v}_{n+1}^{-}, \\
& \text {with : } \quad \tau=h / \Delta t_{n}, \Delta t_{n}=t_{n+1}-t_{n}, h \in(0, \Delta t) .
\end{aligned}
$$

The resulting equation system can be written as (without any further transformation) :

$$
\left(\begin{array}{cccc}
\frac{1}{2} \mathbf{K} & \frac{1}{2} \mathbf{K} & -\frac{1}{3} \Delta t_{n} \mathbf{K} & -\frac{1}{6} \Delta t_{n} \mathbf{K} \\
-\frac{1}{2} \mathbf{K} & \frac{1}{2} \mathbf{K} & -\frac{1}{6} \Delta t_{n} \mathbf{K} & -\frac{1}{3} \Delta t_{n} \mathbf{K} \\
\frac{1}{3} \Delta t_{n} \mathbf{K} & \frac{1}{6} \Delta t_{n} \mathbf{K} & \frac{1}{2} \mathbf{M}+\frac{1}{3} \Delta t_{n} \mathbf{D} & \frac{1}{2} \mathbf{M}+\frac{1}{6} \Delta t_{n} \mathbf{D} \\
\frac{1}{6} \Delta t_{n} \mathbf{K} & \frac{1}{3} \Delta t_{n} \mathbf{K} & -\frac{1}{2} \mathbf{M}+\frac{1}{6} \Delta t_{n} \mathbf{D} & \frac{1}{2} \mathbf{M}+\frac{1}{3} \Delta t_{n} \mathbf{D}
\end{array}\right)\left(\begin{array}{c}
\mathbf{u}_{n}^{+} \\
\mathbf{u}_{n+1}^{-} \\
\mathbf{v}_{n}^{+} \\
\mathbf{v}_{n+1}^{-}
\end{array}\right)=\left(\begin{array}{c}
\mathbf{K} \mathbf{u}_{n}^{-} \\
\mathbf{o} \\
\mathbf{F}_{1}+\mathbf{M v}_{n}^{-} \\
\mathbf{F}_{2}
\end{array}\right)(10
$$


with : $\mathbf{F}_{1}=\int_{\tau}(1-\tau) \mathbf{f}(t) \Delta t_{n} d \tau, \quad \mathbf{F}_{2}=\int_{\tau} \tau \mathbf{f}(t) \Delta t_{n} d \tau$

\subsection{Comparing Discontinuous Galerkin and Newmark type algorithms}

After the description of the general schemes of both time integrators our focus is on the specific features, the disadvantages and advantages. It is well known that essential properties concerning the quality of time integrators can be found through investigations of the amplification matrix $\mathbf{A}\left(\mathbf{A} \in \mathbb{R}^{2 \times 2}\right)$ of a single degree of freedom (SDOF) system. With the eigenvalues $\lambda_{1}, \lambda_{2}$ of $\mathbf{A}$ general statements concerning the spectral stability, algorithmic damping and the phase error (time dispersion) can be developed. For an undamped linear SDOF-system with parameters $m$ (mass) and $k$ (stiffness) the following quantities are obtained:

Phase: $\Omega=\omega \Delta t, \quad$ Spectral radius: $\rho=\max \left|\lambda_{i}\right| ; i=1 \ldots 2$,

Natural frequencies, FE - FD : $\omega_{h}^{I}=\frac{\arctan \frac{\operatorname{Im}(\lambda)}{\operatorname{Re}(\lambda)}}{\Delta t}, \quad \omega_{h}^{I I}=\frac{\frac{\pi}{2}+\arctan \frac{\operatorname{Re}(\lambda)}{\operatorname{Im}(\lambda)}}{\Delta t}$.

$\omega$ is the exact natural frequency, $\omega_{h}$ is the approximated frequency from the time integration scheme. The first equation for $\omega_{h}^{I}(12)$ is valid in the first quadrant of the complex numbers, while the second equation for $\omega_{h}^{I I}$ stands for the second quadrant. The formulas for the other quadrants can be found analogously. There are two bifurcation points for the DG -P2 formulation at $\Omega=3.1$ and $\Omega=10.8$ (visible in figure 3 ). Beyond $\Omega=10.8$ all roots are real, thus the numerical phase remains constant there.

It is known that both the DG and the standard Newmark scheme are 'A' stable, however there are major differences concerning the spectral radii, see figure 3 , and the algorithmic damping. The spectral radius of the Newmark scheme is independent of the phase, whereas the $D G P 1-P 1$ scheme shows a strongly decaying radius for larger phase resp. higher frequencies. The values of the $D G P 2$ scheme, however, do not show a clear dependency, in particular, for the medium range of the phase. Remarkably, the spectral radius remains 1 for the larger phase, thus higher frequency range. This has consequences for the algorithmic damping $\bar{\xi}$ and the phase error $e_{p}$, which are defined as:

$$
\bar{\xi}=-\frac{\ln \rho}{\Delta t \omega_{h}}, \quad e_{p}=\frac{\Omega}{\Omega_{h}}-1, \text { with }: \Omega_{h}=\Delta t \omega_{h} .
$$

Their properties for a wide range of frequencies are shown in figure 4a. resp. 4b. As already visible from figure 3 there is no dependency of the spectral radius from the frequency for the Newmark scheme thus no algorithmic damping over the full range of frequencies. There is a varying dependency of the damping in the low frequency range and no damping of the high frequencies for the $D G P 2$ scheme. A somehow better behavior concerning damping can be found for the $D G P 1-P 1$ method. All high frequencies will be damped out. Thus, the phase error shown in figure $4 \mathrm{~b}$., which causes a dispersion in the time range, will also be damped out, though the phase error in time is growing steadily. It 


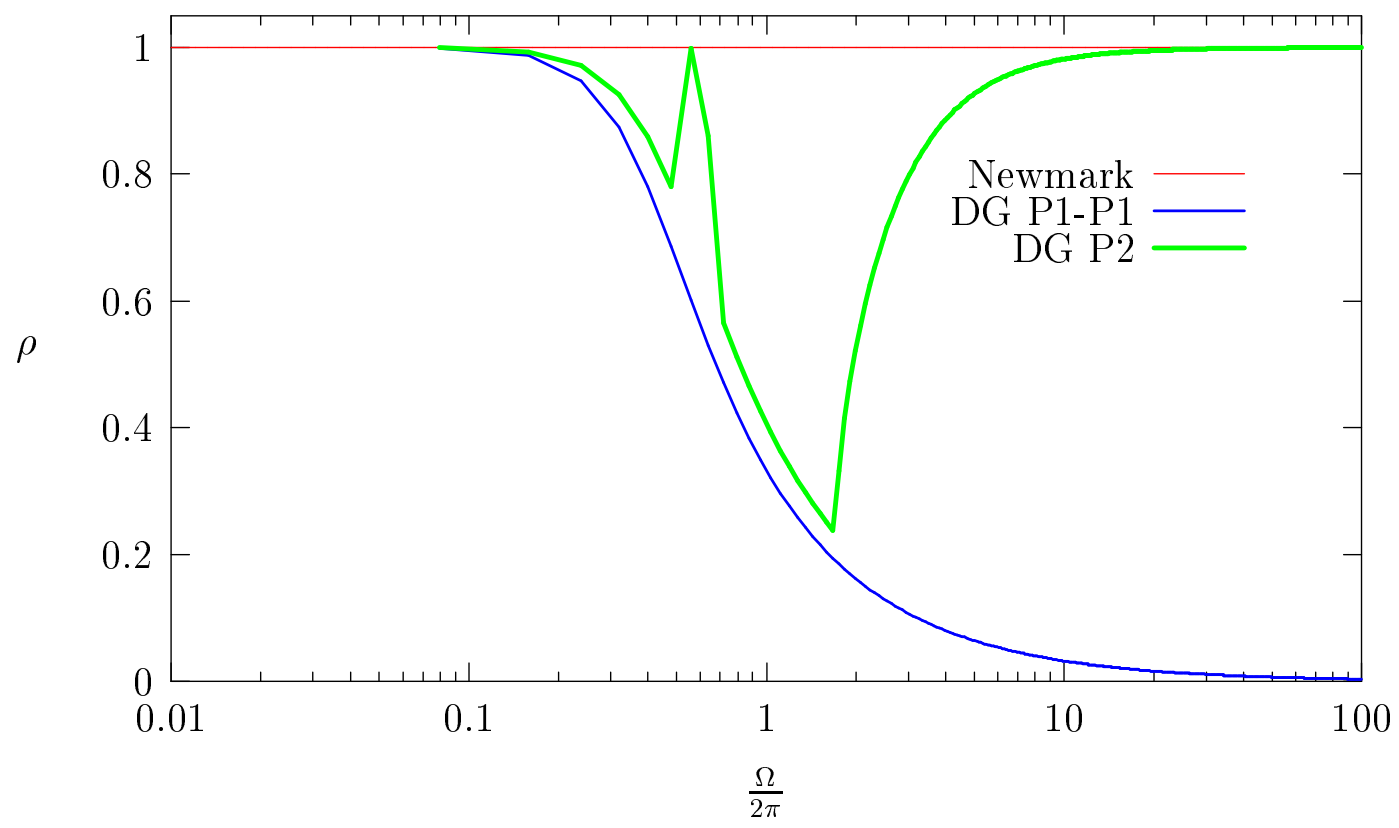

Figure 3: Spectral radii $\rho$ vs. phase for Newmark and DG time integration.

is also remarkable that the phase error of the Newmark scheme remains below the error of the $D G P 1-P 1$ method in the range $\Omega / 2 \pi>1.2$, however, it is not damped out. The phase error of the $D G P 2$ scheme remains well below the error of the Newmark scheme for the full frequency range and similar to the Newmark scheme there is no damping of this error in the high frequency range. Comparing the accuracy of the DG P1-P1 and the $D G P 2$ method by Taylor series expansion we get for the remainder :

$$
D G P 1-P 1: \tau=\frac{1}{36} \mathcal{O}\left(\Delta t^{3}\right), \quad D G P 2: \tau=\frac{1}{72} \mathcal{O}\left(\Delta t^{3}\right) \text {, see Hulbert [11]. }
$$

Thus, there is only little difference in the accuracy. In order to get the artificial dissipation in the upper modes also for the $D G P 2$ scheme least-square operators or so-called shock capturing operators, Hulbert [10] could be used.

Beyond the above mentioned damping properties there are other aspects, which favor the $D G P 1-P 1$ formulation instead of the $D G P 2$ method. First of all, this is the symmetric block-matrix form for the $D G P 1-P 1$ scheme with identical main-diagonals, which is obtained for both $D G$ P1-P1 formulations, Wiberg/Li [16] and Ruge [23], discussed in detail in the following.

Taking the block structure into account Wiberg/Li obtain the following system of four rows of block matrices after a FE discretization:

$$
\left(\begin{array}{cccc}
\mathbf{I} & \mathbf{O} & -\frac{1}{6} \Delta t \mathbf{I} & \frac{1}{6} \Delta t \mathbf{I} \\
\mathbf{O} & \mathbf{I} & -\frac{1}{2} \Delta t \mathbf{I} & -\frac{1}{2} \Delta t \mathbf{I} \\
\mathbf{O} & \mathbf{O} & \mathbf{M}^{*} & \frac{2}{3} \mathbf{M}+\frac{1}{6} \Delta t \mathbf{D} \\
\mathbf{O} & \mathbf{O} & \frac{1}{2} \Delta t \mathbf{D}+\frac{1}{3} \Delta t^{2} \mathbf{K} & \mathbf{M}^{*}
\end{array}\right)\left(\begin{array}{c}
\mathbf{u}_{n}^{+} \\
\mathbf{u}_{n+1}^{-} \\
\mathbf{v}_{n}^{+} \\
\mathbf{v}_{n+1}^{-}
\end{array}\right)=\left(\begin{array}{c}
\mathbf{u}_{n}^{-} \\
\mathbf{u}_{n}^{-} \\
\mathbf{F}_{1}^{*} \\
\mathbf{F}_{2}^{*}
\end{array}\right)
$$

with :

$$
\mathbf{M}^{*}=\mathbf{M}+\frac{1}{2} \Delta t \mathbf{D}+\frac{1}{6} \Delta t^{2} \mathbf{K}, \quad \mathbf{M}^{*} \in \mathbb{R}^{n e q \times n e q}
$$




$$
\mathbf{F}_{1}^{*}=\frac{5}{3}\left(\mathbf{F}_{1}+\mathbf{M} \mathbf{v}_{n}^{-}\right)-\frac{1}{3} \mathbf{F}_{2}-\frac{2}{3} \Delta t \mathbf{K u}_{n}^{-}, \quad \mathbf{F}_{1}^{*}=\mathbf{F}_{1}+\mathbf{F}_{2}+\mathbf{M} \mathbf{v}_{n}^{-}-\Delta t \mathbf{K} \mathbf{u}_{n}^{-}
$$

It is obvious that the third and the fourth row are fully decoupled from the first two rows,

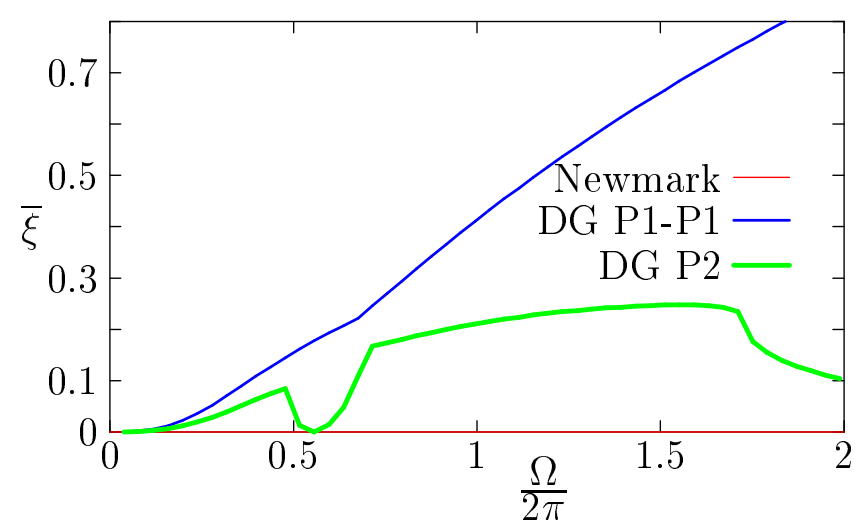

a.)

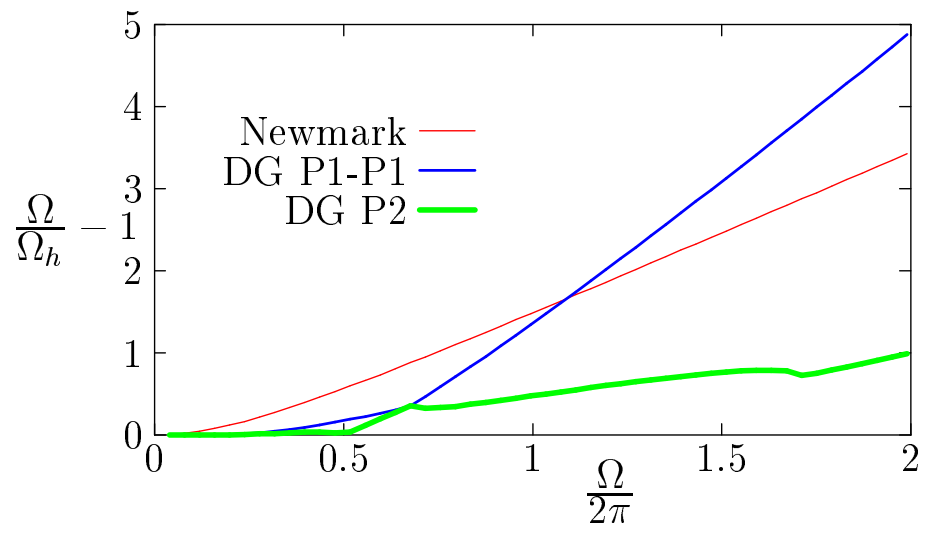

b.)

Figure 4: Algorithmic damping resp. phase error vs. phase $\Omega / 2 \pi$ for Newmark and DG integration schemes.

thus only a system of $2 \times n e q$ equations remains. Furthermore, this large non-symmetric equation system can be favorably solved by Gauss-Seidel block iteration. For an efficient iterative solution the spectral radius of the iteration matrix should be small, which may not be the case for some problems in general. The iterative solution for the velocities is then written as, see Wiberg/Li [16]:

Iteration $i$ :

$$
\begin{aligned}
& \mathbf{M}^{*} \mathbf{v}_{n}^{+(i)}=\mathbf{F}_{1}^{*}-\left(\frac{2}{3} \mathbf{M}+\frac{1}{6} \Delta t \mathbf{D}\right) \mathbf{v}_{n+1}^{-(i-1)} \\
& \mathbf{M}^{*} \mathbf{v}_{n+1}^{-(i)}=\mathbf{F}_{2}^{*}-\left(\frac{1}{2} \Delta t \mathbf{D}+\frac{1}{3} \Delta t^{2} \mathbf{K}\right) \mathbf{v}_{n}^{+(i)}
\end{aligned}
$$

Convergence check with:

$$
\operatorname{diff}=\frac{\left|\left(\begin{array}{c}
\mathbf{v}_{n}^{+(i)} \\
\mathbf{v}_{n+1}^{-(i)}
\end{array}\right)-\left(\begin{array}{c}
\mathbf{v}_{n}^{+(i-1)} \\
\mathbf{v}_{n+1}^{-(i-1)}
\end{array}\right)\right|}{\left|\left(\begin{array}{c}
\mathbf{v}_{n}^{+(i)} \\
\mathbf{v}_{n+1}^{-(i)}
\end{array}\right)\right|}
$$

if $\operatorname{diff} \leq$ tol then finish; $\quad$ else continue with next iteration cycle.

The advantage of the iterative block solution in the manner shown above is that the $L D L^{T}$ decomposition of the matrix $\mathbf{M}^{*}$ has to be performed only once. Thus, only forward - backward substitutions are required for the solution in each iteration cycle. After convergence of the solution for the velocities the displacements can be computed without much effort from the two remaining sets of equations, row one and two:

$$
\mathbf{u}_{n+1}^{-}=\mathbf{u}_{n}^{-}+\frac{1}{2} \Delta t\left(\mathbf{v}_{n}^{+}+\mathbf{v}_{n+1}^{-}\right), \quad \mathbf{u}_{n}^{+}=\mathbf{u}_{n}^{-}+\frac{1}{6} \Delta t\left(\mathbf{v}_{n}^{+}-\mathbf{v}_{n+1}^{-}\right) .
$$


An alternative to the scheme of Wiberg/Li is suggested by Ruge [23] based on the variation of the scalar integral $I$, where $H=T+U$, kinetic plus strain energy, is the Hamiltonian:

$$
U=\frac{1}{2} \int_{\Omega}(\mathbf{B} \mathbf{u})^{T} \mathbf{C} \mathbf{B u} d V, \quad T=\frac{1}{2} \int_{\Omega} \mathbf{p}^{T} \rho^{-1} \mathbf{p} d V, \quad \mathbf{p}=\rho \mathbf{v} .
$$

Taking the potential $W_{a}$ of the external forces into account, the integral $I$ over the time interval $\Delta t$ is obtained as:

$$
I=\int_{\Delta t}\left(H+\Pi_{a}\right) d t, \quad \Pi_{a}=-\int_{\Omega} \mathbf{u}^{T} \mathbf{p} d V .
$$

The standard requirement concerning $I$ (referring to Hamilton's principle) is, that it remains stationary : $\delta I=0$. By variation and partial integration with respect to time we obtain :

$$
\delta I=\int_{\Delta t} \int_{\Omega} \delta \mathbf{p}^{T}\left[\dot{\mathbf{u}}-\frac{\partial H}{\partial \mathbf{p}}\right] d \Omega d t-\int_{\Delta t} \delta \mathbf{u}^{T}\left[\dot{\mathbf{p}}+\frac{\partial U}{\partial \mathbf{u}}-\mathbf{f}\right] d t=0 .
$$

Usually this formulation leads to a conditionally stable integrator with an order of accuracy $\mathcal{O}(\Delta t)$, if $\mathbf{u}, \mathbf{v} \in P^{1}(t) ; t \in\left[t_{n}, t_{n+1}\right]$. To overcome the conditional stability, Ruge [23], following Aharoni/ Bar-Yoseph [1], proposed to add the work of the state variables at the beginning of the time step to the integral, thus introducing them as independent variables. Then the variation $\delta \mathbf{u}_{n}^{+}$and $\delta \mathbf{v}_{n}^{+}$are now also not zero leading to the following form :

$$
\delta I^{*}=\delta I+\rho \int_{\Omega} \delta \mathbf{v}_{n}^{+}\left[\mathbf{u}_{n}^{+}-\mathbf{u}_{n}^{-}\right] d V-\rho \int_{\Omega} \delta \mathbf{u}_{n}^{+}\left[\mathbf{v}_{n}^{+}-\mathbf{v}_{n}^{-}\right] d \Omega=0 .
$$

With linear test functions $\mathbf{u}(t)$ and $\mathbf{v}(t)$ - a P1-P1 scheme - the following non-symmetric set of equations is developed:

$$
\begin{array}{r}
\left(\begin{array}{rr}
\frac{1}{\Delta t} \mathbf{M}+\frac{2}{3} \mathbf{D}+\frac{5}{18} \Delta t \mathbf{K} & -\frac{\Delta t}{6} \mathbf{D}-\frac{\Delta t^{2}}{9} \mathbf{K} \\
-\frac{1}{\Delta t} \mathbf{M}+\frac{2}{3} \mathbf{D}+\frac{7}{18} \Delta t \mathbf{K} \quad \mathbf{M}+\frac{\Delta t}{6} \mathbf{D}-\frac{\Delta t^{2}}{18} \mathbf{K}
\end{array}\right)\left(\begin{array}{c}
\mathbf{u}_{n+1}^{-} \\
\mathbf{v}_{n+1}^{-}
\end{array}\right)= \\
\left(\begin{array}{cc}
\frac{1}{\Delta t} \mathbf{M}+\frac{2}{3} \mathbf{D}-\frac{2}{9} \Delta t \mathbf{K} & \mathbf{M} \\
-\frac{1}{\Delta t} \mathbf{M}+\frac{1}{3} \mathbf{D}-\frac{1}{9} \Delta t \mathbf{K} & \mathbf{O}
\end{array}\right)\left(\begin{array}{c}
\mathbf{u}_{n}^{-} \\
\mathbf{v}_{n}^{-}
\end{array}\right)+\left(\begin{array}{c}
\mathbf{f}_{1} \\
\mathbf{f}_{2}
\end{array}\right) \\
\text { Written in short form as : } \mathbf{A} \mathbf{z}_{1}=\mathbf{B} \mathbf{z}_{0}+\mathbf{r} .
\end{array}
$$

This system can be transformed to a similar structure with identical block diagonals as for the rows 3 and 4 of the Wiberg-Li scheme. With specifically chosen hyper-matrices the following system of equations is found:

$$
\begin{aligned}
& \mathbf{T}_{1}=\left(\begin{array}{cc}
\mathbf{0} & \mathbf{1} \\
-\mathbf{1} & \mathbf{1}
\end{array}\right), \quad \mathbf{T}_{2}=\left(\begin{array}{cc}
\mathbf{1} & \mathbf{1} \\
\frac{2}{\Delta t} \mathbf{1} & \frac{3}{\Delta t} \mathbf{1}
\end{array}\right), \text { with eq. (19) and } \mathbf{z}_{1}=\mathbf{T}_{2} \mathbf{y}_{1} \\
& \mathbf{T}_{1} \mathbf{A} \mathbf{T}_{2} \mathbf{y}_{1}=\mathbf{T}_{1} \mathbf{B} \mathbf{z}_{0}+\mathbf{T}_{1} \mathbf{r}, \quad \rightarrow \mathbf{A}^{*} \mathbf{y}_{1}=\mathbf{B}^{*} \mathbf{z}_{0}+\mathbf{r}^{*} \\
& \text { with } \mathbf{A}^{*}=\left(\begin{array}{cc}
\frac{\mathbf{M}}{\Delta t}+\frac{2}{3} \mathbf{D}+\frac{5}{18} \Delta t \mathbf{K} & 2 \frac{\mathbf{M}}{\Delta t}+\frac{5}{6} \mathbf{D}+\frac{2}{9} \Delta t \mathbf{K} \\
\frac{\mathbf{D}}{3}+\frac{2}{9} \Delta t \mathbf{K} & \mathbf{A}_{11}^{*}
\end{array}\right) .
\end{aligned}
$$


Then the same iteration procedure with a block Gauss-Seidel scheme as described for the Wiberg/Li equation system can be used .

However, the major difference between both equation systems is that Ruge's scheme contains the variables $\mathbf{u}_{n+1}^{-}$and $\mathbf{v}_{n+1}^{-}$, whereas Wiberg/Li solve for the velocities $\mathbf{v}_{n}^{+}$and $\mathbf{v}_{n+1}^{-}$directly. Thus, a direct comparison is difficult and the judgment is preferably based on the efficiency of the solution. The latter is mainly influenced by the condition number of the system matrices, which can be decomposed as follows :

$$
\mathbf{A} \text { resp. } \mathbf{A}^{*}=-\mathbf{L}+\mathbf{S}-\mathbf{U},
$$

S ... diagonal matrix of $\mathbf{A}$ (13) resp. $\mathbf{A}^{*}$ (22); $\mathbf{L}, \mathbf{U}$... lower resp. upper triangular matrix of matrix $\mathbf{A}$ resp. $\mathbf{A}^{*}$.

A comparison shows that the diagonal of matrix $\mathbf{A}$ (eq. (13)) of the Wiberg scheme is far more dominant than the diagonal of matrix $\mathbf{A}^{*}$ (eq. (22)). In figure 5 the spectral radius $\rho_{i t}$ of the respective iteration matrix $\mathbf{T}_{i t}=(\mathbf{S}-\mathbf{L})^{-1} \mathbf{U}$ is presented for both integration schemes applied to a SDOF with varying stiffness. It is obvious that the spectral radius is much smaller for Wiberg/Li's scheme resulting in a better convergence. Furthermore, an attempt was made to improve the spectral radius by a combination with a relaxation scheme. Then the modified iteration matrix $\mathbf{T}_{i t}$ becomes:

$$
\mathbf{T}_{i t}=(\mathbf{S}-\eta \mathbf{L})^{-1}[(1-\eta) \mathbf{S}+\eta \mathbf{U}]
$$

In the example (SDOF) the relaxation parameter $\eta$ was varied between $0.95,1.00$ and 1.1. For the chosen system parameters a considerable improvement in the spectral radius was found for $\eta=1.1$. However, in real systems with a widespread band of frequencies no visible advantage using such a relaxation scheme could be achieved.

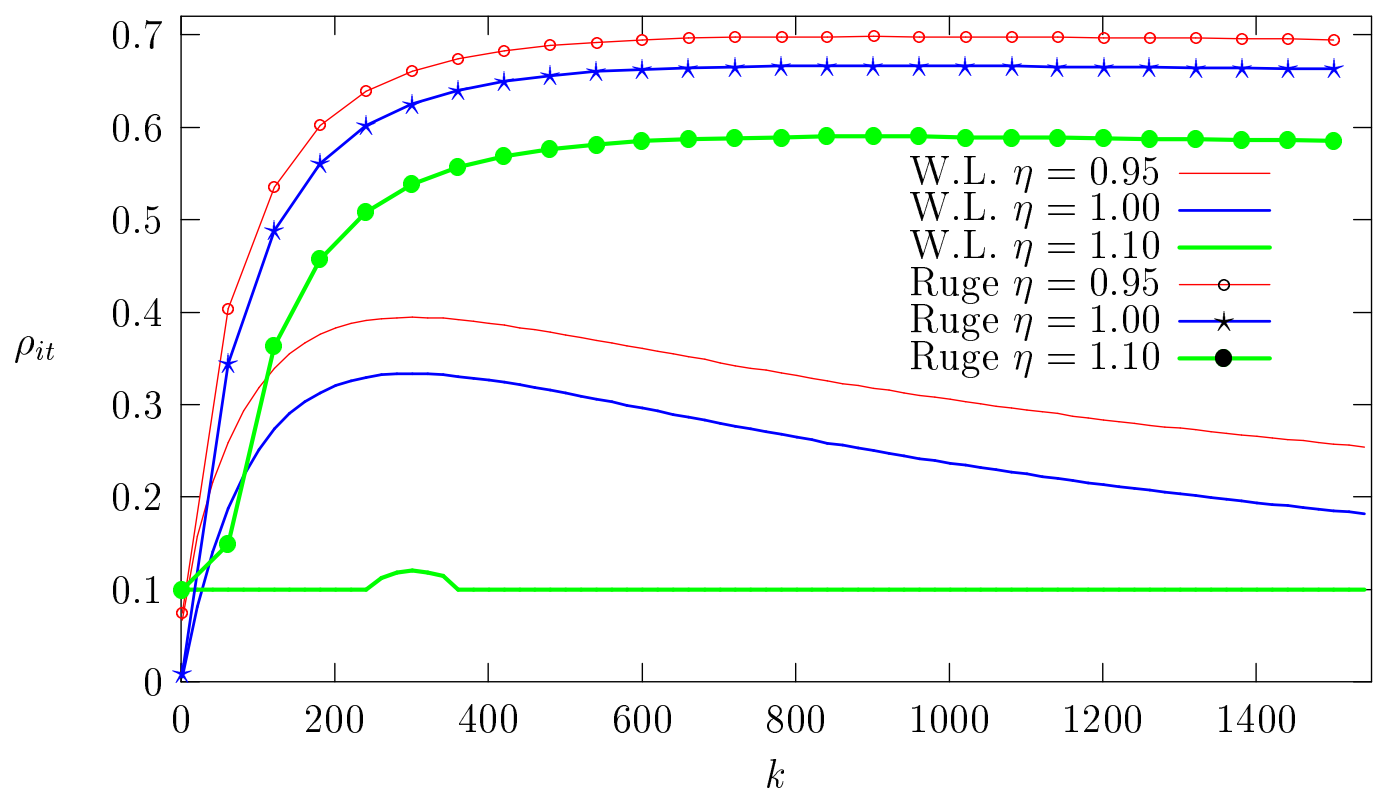

Figure 5: Spectral radius $\rho_{i t}$ vs. stiffness $k$ for the $D G P 1-P 1$ schemes proposed by Wiberg/Li and Ruge combined with relaxation;

SDOF system: $m=0.5=$ const., $\Delta t=0.1=$ const . 
Finally, the wave propagation in a slab, see figure 6 , is considered to compare the performance of the solution schemes without relaxation using a constant time step size and a constant mesh. From figure 7 it can be concluded that the effort required for Ruge's scheme is considerably higher at least in the beginning of the process. Similar observations have been made for other problems.

\section{Comparing the performance in time of DG and Newmark time integration for wave propagation and vibration problems}

In order to show the difference between a DG and the standard Newmark method two completely different types of problems are investigated; an example of an instationary problem and one for stationary motion. A classical example for an instationary problem is the wave propagation in solids, while the vibration of a cantilever plate represents a stationary vibration problem. For both cases different parts of the frequency spectrum are dominantly excited.

In the wave propagation problem, see figure 8, the response is in the complete frequency range, whereas in the stationary problem predominantly the response is in the lower frequencies. In the left diagram in figure 8 the time history for the total energy norm of the solution $\|\mathbf{u}\|$ is presented. The dissipative property of the $D G P 1-P 1$ is obvious. About $25 \%$ of the energy is dissipated after 80 time units, whereas the energy is perfectly conserved for the Newmark scheme. The comparison of the velocity component $v_{x}$ shows the positive aspects of the $D G P 1-P 1$ scheme on the other hand. As a result of the algorithmic damping, the higher (less accurate) frequencies are filtered out and a smooth response is achieved. It is also obvious that both schemes cannot maintain the correct velocity amplitudes. Although the Newmark scheme conserves the energy, the velocity amplitude shows a strong variation due the inherent spatial dispersion of the FEM-mesh. The conclusion is rather clear: Both the DG P1-P1 and Newmark are not applicable for long time integration in such a case. However, for short duration problems the DG P1-P1 scheme performs rather well.

The second case, a stationary motion of a clamped cantilever plate with a line load at the free end - see figure 9 - is investigated next. In the diagrams in figure 10 the total energy norm of the solution and the vertical velocity $v_{z}$ at the free end are presented. As expected there is hardly any difference between the results obtained with both schemes. Obviously higher frequencies are not excited and there is no accuracy advantage for any method. However, the solution effort needed for the Newmark scheme is far below the effort required for any DG scheme for such a problem and it should be preferred then.

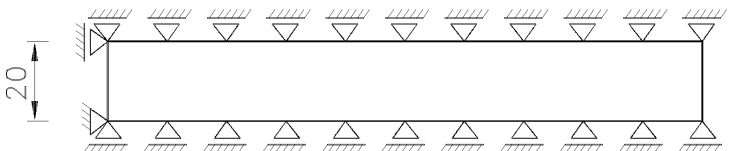

200
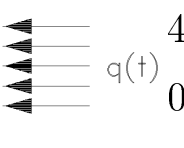

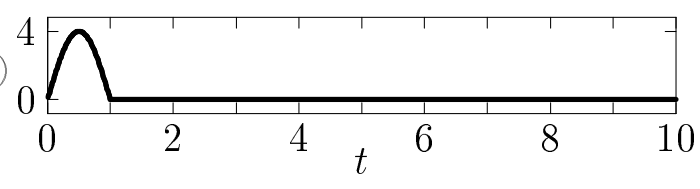

Figure 6: Wave propagation problem; system and load function vs. time;

Material parameter: $E=1 \cdot 10^{4}, \rho=10, t=1, \nu=0.0$. 


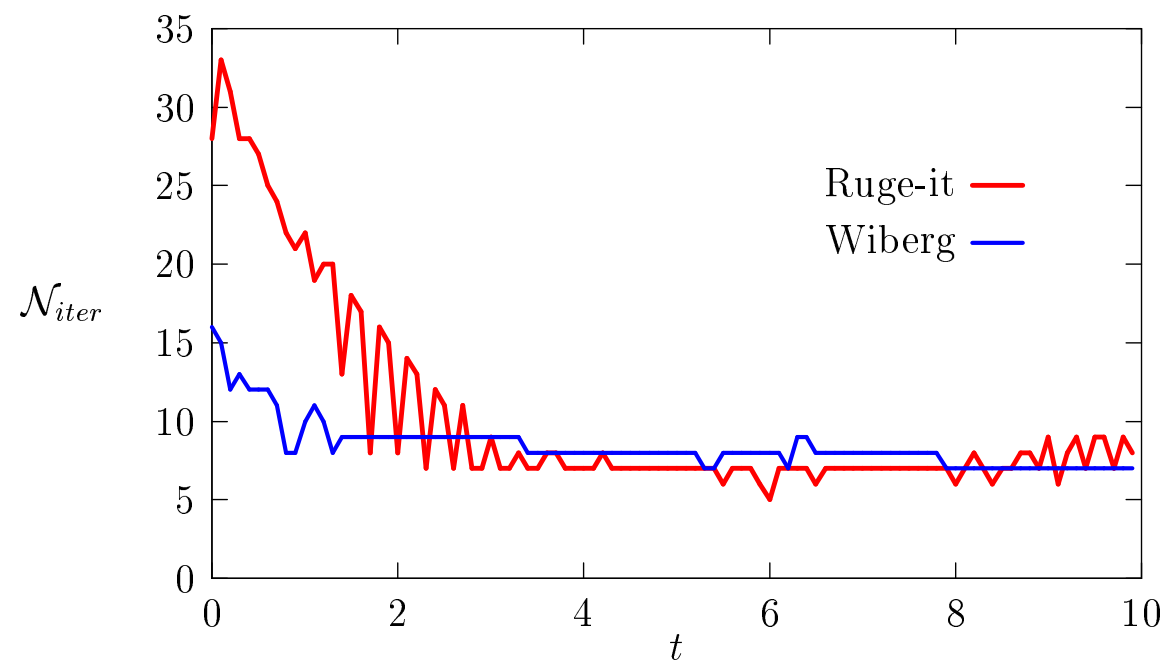

Figure 7: Number of Iterations $\mathcal{N}_{i t}$ for $D G$ P1-P1 methods; Example - wave propagation.

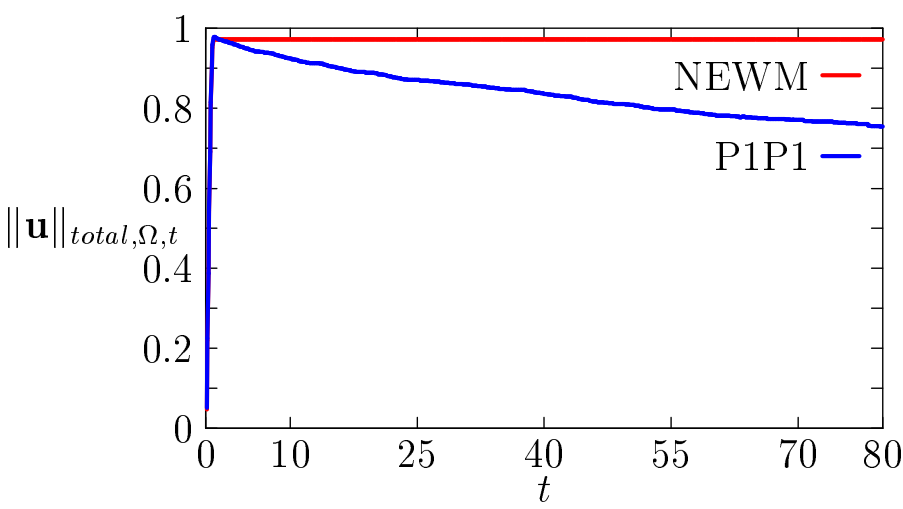

a.)

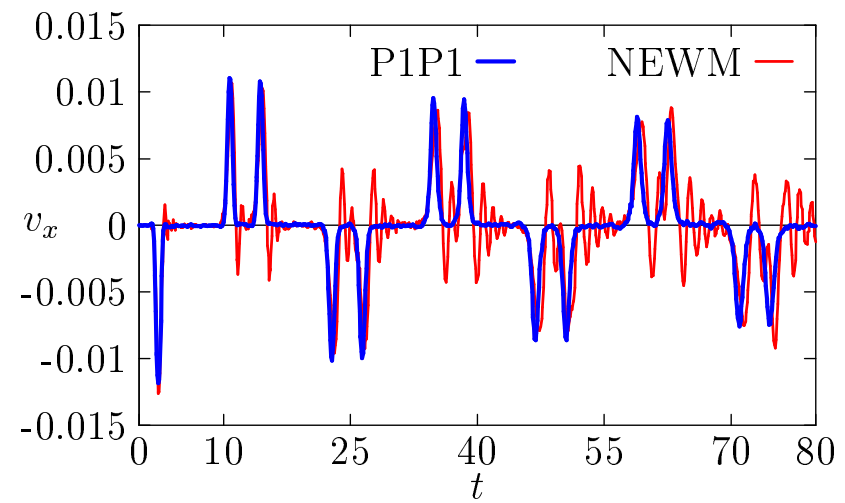

b.)

Figure 8: Wave propagation problem - Time history plot of a.) total energy norm $\|\mathbf{u}\|_{\text {total }, \Omega}$ and b.) velocity $v_{x}$ at $x=140$; Comparison of Newmark and $D G$ P1-P1 schemes.
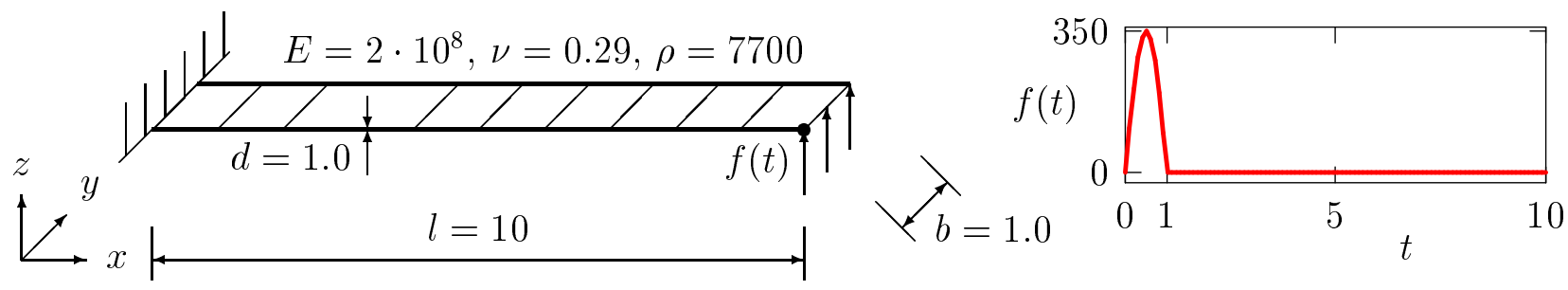

Figure 9: Clamped plate with line load; example for stationary motion.

\section{Dispersion effects depending on the spatial FE - discretization}

\subsection{General}

The time integration schemes discussed above showed some phase error (eq. (12), thus some dispersion changing in time was already found. A similar effect is introduced by the 


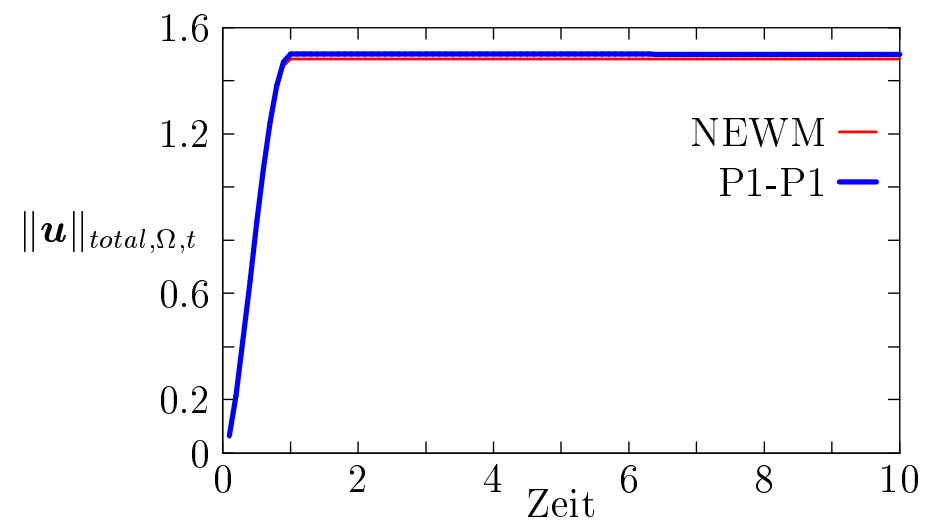

a.)

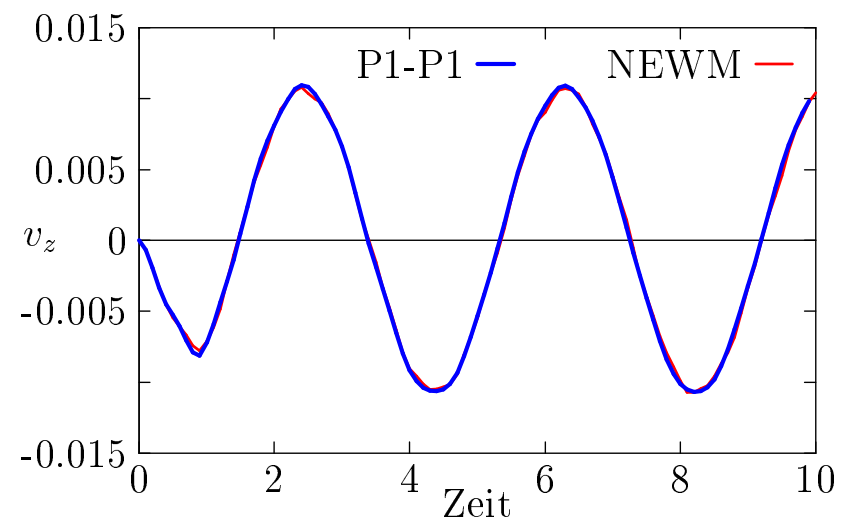

b.)

Figure 10: Clamped plate, stationary motion problem - Time history plot of a.) total energy norm $\|\mathbf{u}\|_{\text {total }, \Omega}$ and b.) velocity $v_{z}$ at end of plate; Comparison of Newmark and $D G P 1-P 1$ time integration.

spatial discretization. This artificial dispersion, however, has to be separated from any dispersion which is inherent in the problem described by the corresponding differential equation. Such phenomena can be investigated rather well in wave propagation problems. There, every wave length $\lambda_{i}$ has its own propagation velocity $c\left(\lambda_{i}\right)$.

In figure 11a.) the inherent dispersion for the wave propagation of a bending mode in plates is shown depending on the kinematic model chosen [18]. Every model (Mindlin plate, Kirchhoff plate and 3D continuum) has its own dispersion characteristics which are discussed briefly in the following with the definitions:

$h \ldots$ thickness of the plate, $\lambda \ldots$ wave length of the bending wave, $c \ldots$ propagation velocity of the bending wave, $c_{s} \ldots$ exact propagation velocity of the shear wave; $c_{s}=\sqrt{\frac{E}{2(1+\nu) \rho}}$.

From figure 11a. it is obvious that the Kirchhoff model is able to represent the bending waves only for large wave lengths resp. low wave numbers, and the kinematic model of Mindlin corresponds very well to the exact solution of the general 3D model for the full range of wave numbers. Comparing the longitudinal resp. membrane waves for the 3D continuum and 1D continuum e.g. a bar with circular cross section, a significant dispersion is found for the exact solution of the 3D case with the definitions :

$c_{o}=\sqrt{E / \rho} \ldots$ propagation velocity of $1 \mathrm{D}$ continuum,

$\bar{c} \ldots$ propagation velocity of longitudinal wave; $\bar{\lambda}$... wave length of longitudinal wave .

However, it is also clear that the simplest reduced continuum model, the $1 \mathrm{D}$ case is able to represent the exact solution of the 3D case $(c=c(\lambda))$ only for large wave lengths. For the longitudinal waves in figure $11 \mathrm{~b}$. the exact dispersion in $3 \mathrm{D}$ converges to a constant value; here: $\lim _{\bar{\lambda} \rightarrow 0} c(\overline{\lambda)}=0.57$.

Now the nondispersive characteristics of the $1 \mathrm{D}$ wave propagation problem are used to investigate the artificial dispersion due to the spatial discretization. The well known linear homogeneous differential equation for the 1D wave propagation problem in a bar with a constant cross section is - assuming for completeness some boundary and initial conditions:

$$
\rho \frac{\partial^{2} u}{\partial t^{2}}-E \frac{\partial^{2} u}{\partial x^{2}}=0
$$




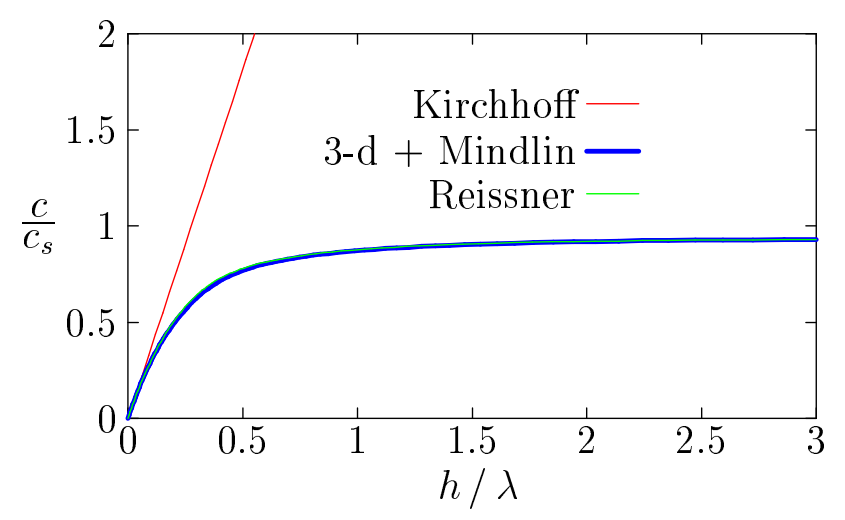

a.) Bending wave in plates [18]

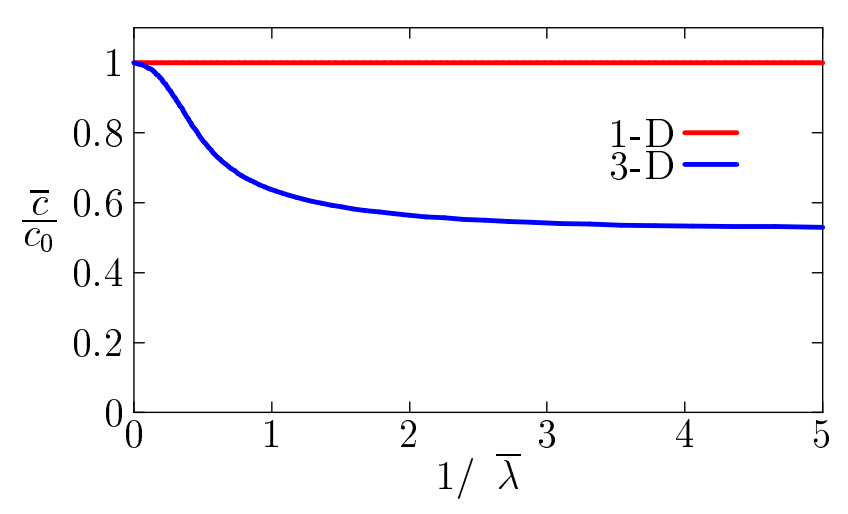

b.) Longitudinal waves in $1 \mathrm{D}$ and $3 \mathrm{D}$ continuum after Graff [5]

Figure 11: Propagation velocity vs. inverse of wave-length.

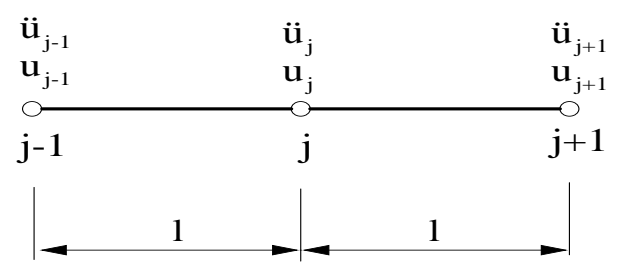

Figure 12: Discretized bar; unknown quantities at nodes.

For the solution the standard assumption is taken: $u(x, t)=A \exp i \omega(x / c-t)$.

Without loss of generality the following can be used as test and trial functions for discretization in space:

$$
\text { test space: } v(x) \in H_{0}^{1}(\Omega), \quad \text { trial space: } u(x) \in H^{1}(\Omega) \text {. }
$$

With the standard Bubnov-Galerkin method, weighting with the test-functions and using Gauss-Green's theorem the ordinary differential equation for node $j$ (see Fig. 12) in a FE mesh with uniform element length $l$ is obtained :

$$
\ddot{u}_{j+1}+4 \ddot{u}_{j}+\ddot{u}_{j-1}+6 \frac{c_{0}^{2}}{l^{2}}\left(-u_{j+1}+2 u_{j}-u_{j-1}\right)=0, \quad j \ldots \text { index for nodes } .
$$

Using the same assumption as for the exact solution: $\quad u_{j}=C \exp i\left(\omega_{h} t-a j\right)$, with:

$C \ldots$ constant to fulfill the initial conditions, $\omega_{h} \ldots$ circular frequency, $\lambda \ldots$ wave length, $a \ldots$ normalized wave number; $a=\frac{2 \pi}{\lambda}$,

the following relation between the normalized wave number $a$ and the circular frequency $\omega_{h}$ for the discretized solution is found, see Nilsson[20]:

$$
\omega_{h}=\frac{c_{0}}{l} \sqrt{6 \frac{1-\cos a}{2+\cos a}} .
$$

Contrary to the analytical exact solution the approximate solution has a so-called cutoff frequency $\omega_{c o}$, the maximum frequency which can be represented in the discretized 


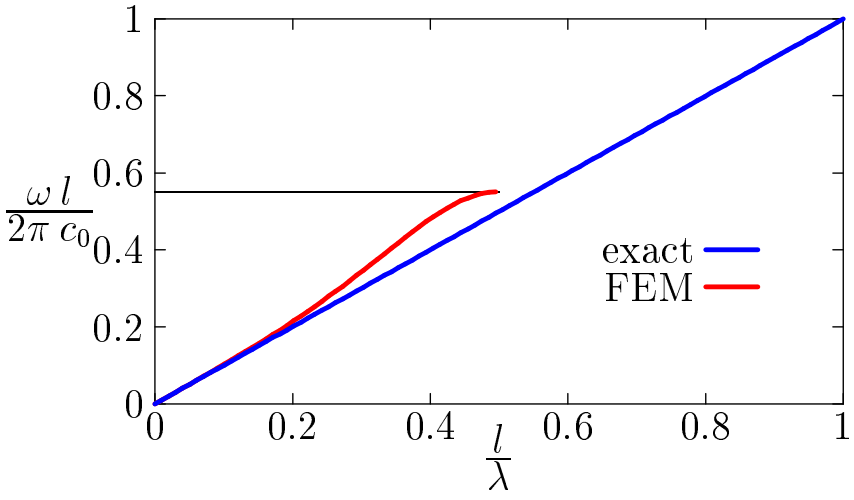

a.) Circular frequency with cut off

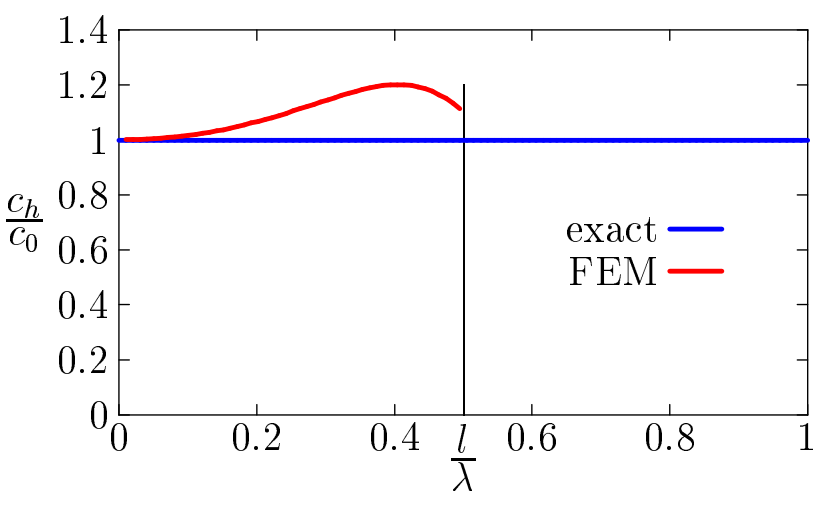

b.) propagation velocity with cut off

Figure 13: Circular frequency resp. propagation velocity vs. ratio of element length/ wave length of discretized 1D continuum.

system, see figure 13a. Thus, using a fixed $l$ for an element, one can only find a maximum frequency $\omega_{c o}$ corresponding to a constant ratio $l / \lambda$. As a consequence of this nonlinear wave-length-frequency relation a non-constant propagation velocity $c_{h}$, which also depends on the wave-length, is obtained:

$$
c_{h}=\frac{\lambda_{h}}{T_{h}}=\frac{\omega_{h} l}{a} .
$$

Thus, it can be concluded that even for the most simple problem without dispersion the corresponding FE-discretization introduces artificial dispersion. It is obvious that a strict distinction between artificial and physical dispersion is not possible for $2 \mathrm{D}$ and $3 \mathrm{D}$ continua.

\subsection{Uniform discretization in space}

In order to show the combined effect of space and time discretization, a wave propagation example, the $1 \mathrm{D}$ continuum problem as shown in figure 6 , is investigated. The mesh is kept uniform and unmodified during the analysis. Three types of time integration are compared: First, the fully exact solution in time and space is given by an analytical solution. Second, the structure is discretized in space by FE and followed by modal decomposition. Then each modal response is integrated separately and summed up exactly as modal solution. As third action the FE discretization in space is combined with either FET (DG P1-P1) resp. FD (Newmark) in time. In the following diagrams the stress state $\sigma(x)$ of the whole space domain is plotted at several times.

As expected, see figure 14, the modal solution is somehow in front of the exact solution because of the artificial dispersion inherent in the discretized continuum. The short waves have a higher propagation velocity than the long waves causing the waviness in front of the sinusoidal half-wave. With progressing time this artificial dispersion increases. The Newmark solution shows some artificial waves traveling behind the exact and the modal 


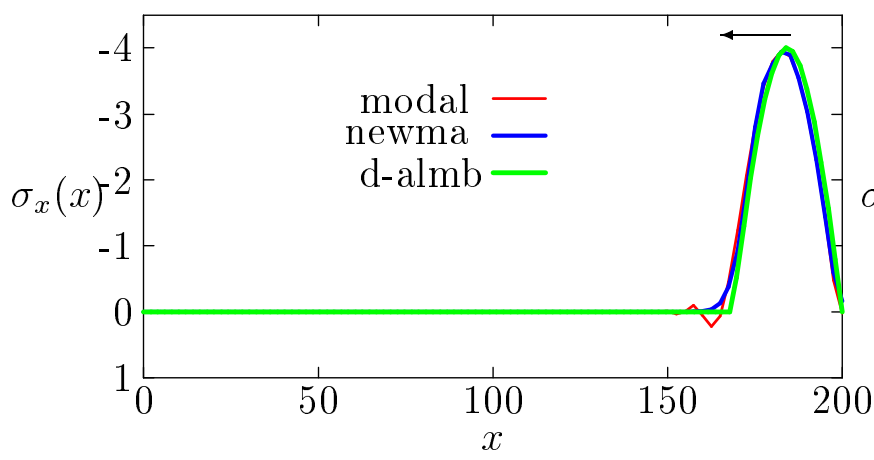

a.) $t=1.0$

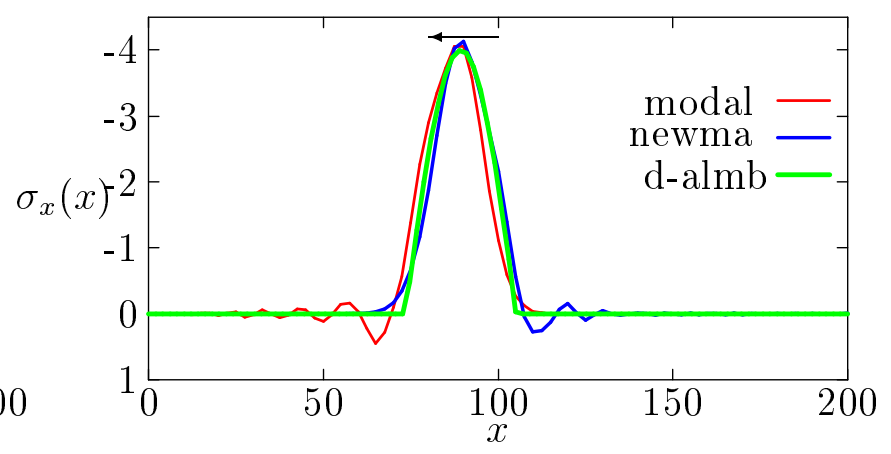

b.) $t=4.0$

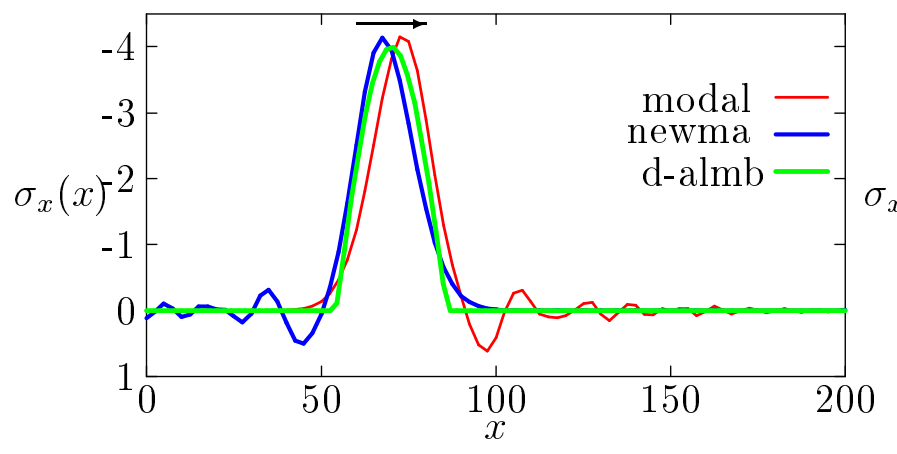

c.) $t=9.0$

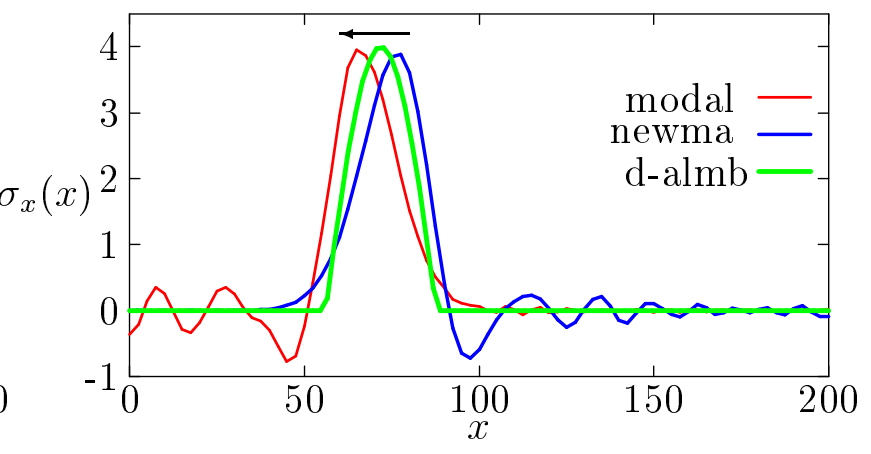

d.) $t=17.2$

Figure 14: 1D wave propagation - Stress state of complete space domain at different time states; uniform mesh; behavior of Newmark scheme.

solution. This is mainly due to the phase error (see fig. 4b.) which accumulates in time. However, it may be noted that taking a much smaller time step, a similar solution as for the modal method could be obtained.

For the DG P1-P1 method, see figure 15, the modal solution is approximated rather well mainly due to the order of accuracy $\mathcal{O}\left(\Delta t^{3}\right)$ of the integration scheme. The effect of the phase error seems to be small for this type of excitation which is also due to the artificial damping of the higher frequencies. The latter also leads to some decay of the major amplitude and to reduced amplitudes of the small waves running in front of the sinusoidal large wave.

\subsection{Adaptive discretization in space}

If instationary meshes are used, e.g. adaptive mesh generation takes place, then the situation changes. In the following for the estimation of the spatial discretization error the well-known estimator of Zienkiewicz/Zhu [29] for the strain energy norm of error and an additional indicator for the kinetic energy part, proposed by Riccius, Schweizerhof [22] are applied. In order to judge the effect of the time integration schemes the time step is kept constant. Thus, the exact analytical solution and fully numerical solutions are compared. Due to the graded meshes, generated in the adaption process, the maximum amplitude in the response $\sigma_{x}$ presented in figure 16 at various time states is considerably reduced for both numerical schemes. Though the artificial dispersion cannot be controlled perfectly, 


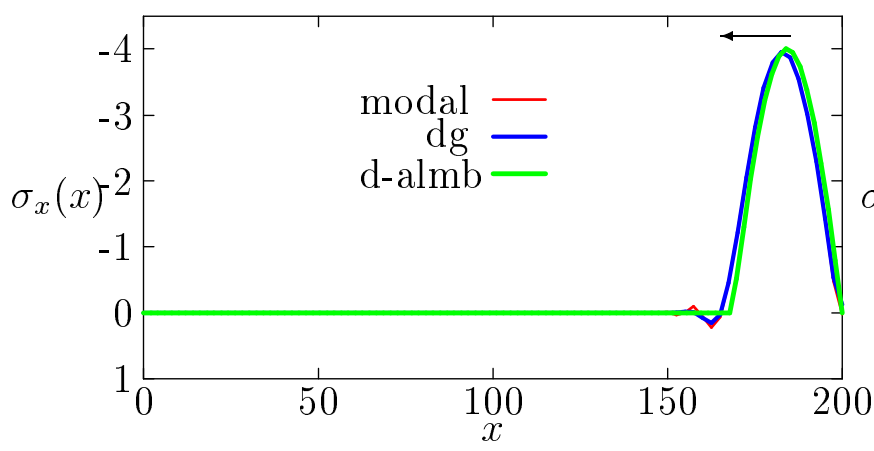

a.) $t=1.0$

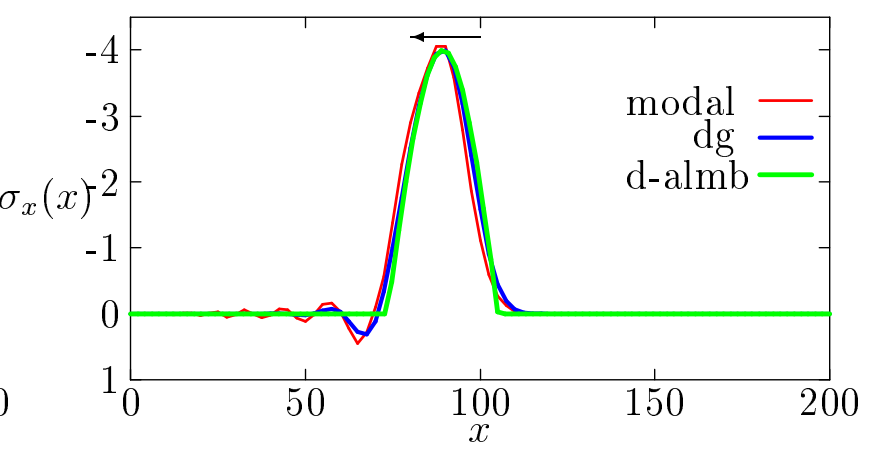

b.) $t=4.0$

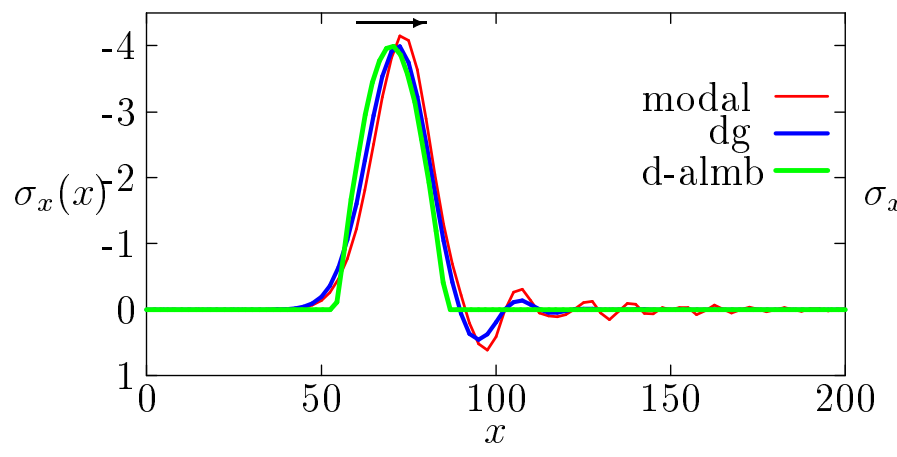

c.) $t=9.0$

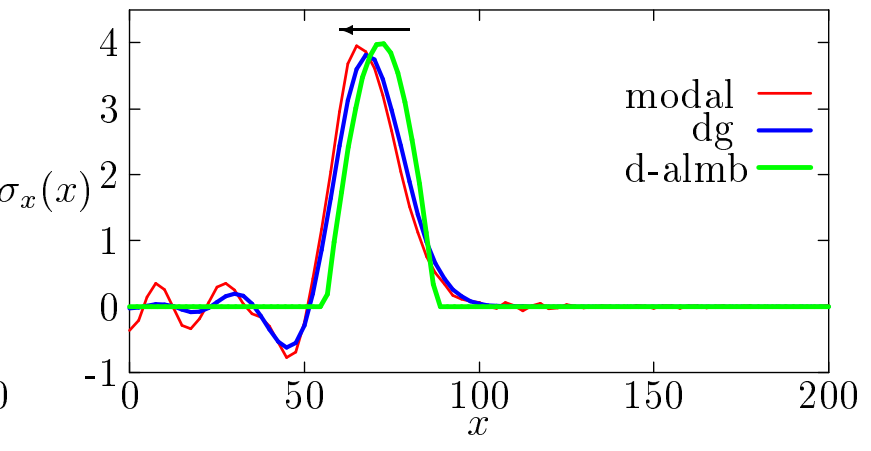

d.) $t=17.2$

Figure 15: 1D wave propagation - stress state of complete space domain at different times; uniform mesh; behavior of $D G P 1-P 1$ scheme.

very good results can be achieved with the $D G P 1-P 1$ method. Only a small increase of the width of the wave and no longer additional small waves are obtained. Furthermore, there is only a very small phase error visible after a while, e.g. at time $t=17.2$. However, the solution errors with the Newmark method increase considerably with time. In particular, the artificial wave following the physical wavefront grows. The reason is the mesh refinement in space also for the region of these smaller waves.

An amplitude decay is visible for both methods. For the energy conserving Newmark scheme this is caused by the energy transfer from the long wave to the smaller artificial waves. The latter is also the reason for the energy decay - by far less pronounced - in the $D G$ P1-P1 method, as it is not possible to damp out the higher modes without affecting the lower modes. Thus, the artificial damping of the DG P1-P1 scheme will damp out all modes in time as $t \rightarrow \infty$. Typical meshes created in the adaptive process are shown in figure 18 .

\section{Spurious Reflections}

Besides the discussed effect of artificial dispersion the adaptive meshing could also create so-called spurious reflections. Bazant/Celep [2] investigated this phenomena on stationary graded meshes with the conclusions that only short waves will be reflected at the element interface in the case $l_{2} \geq 2 l_{1}$, see figure 17 , and $l_{1} \geq \lambda / 10$, if a graded mesh is created. However, such an effect was not observed during all investigations with instationary 


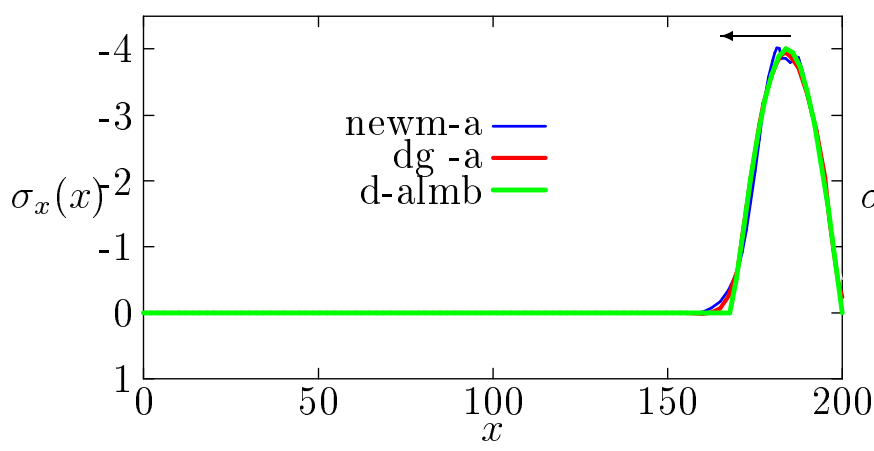

a.) $t=1.0$

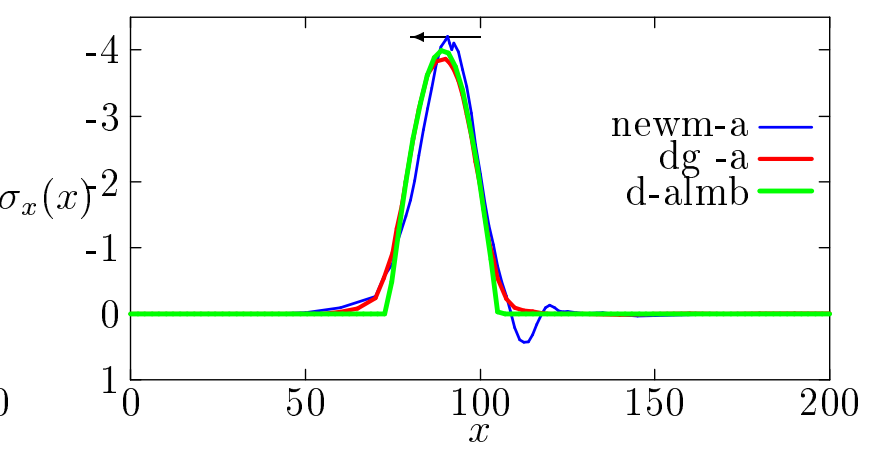

b.) $t=4.0$

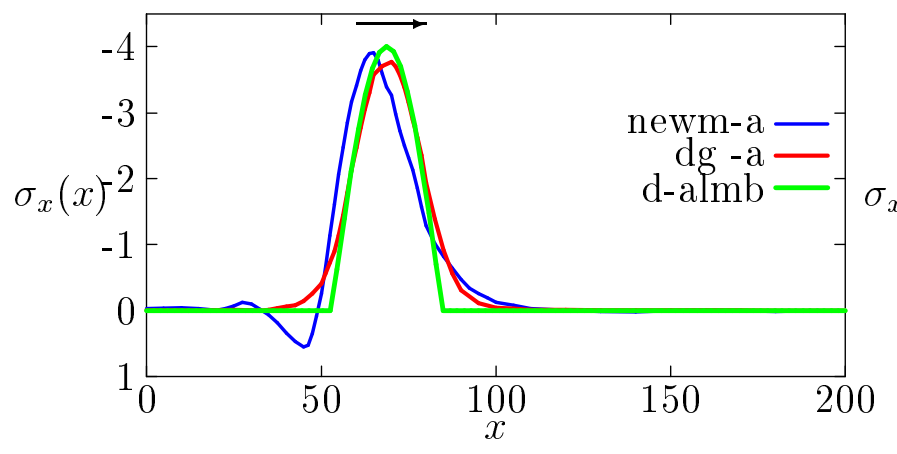

c.) $t=9.0$

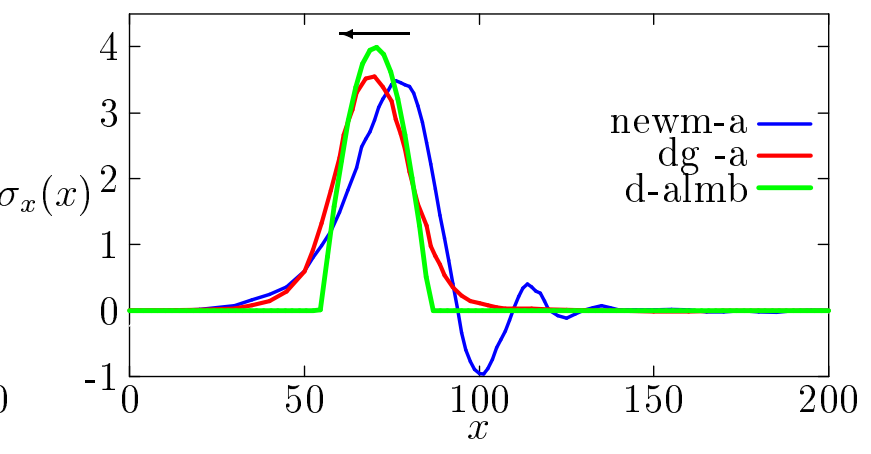

d.) $t=17.2$

Figure 16: 1D wave propagation - stress state of complete space domain; comparing DG P1-P1 and Newmark time integration with exact solution; adaptive discretization in space.

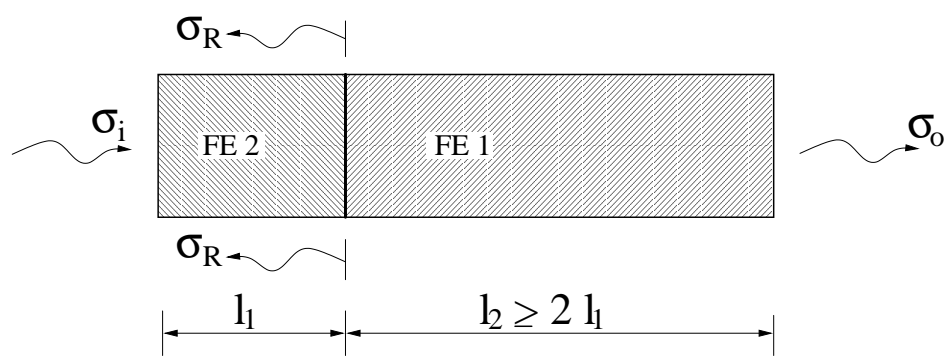

Figure 17: Spurious reflections in graded meshes.

(adaptive) meshes so far.

\section{Adaptive time stepping}

Though in general it is possible to integrate the complete time dependent problem with e.g. time-space finite elements with different time step size for each spatial element, see Hulbert/Hughes [9] or at least parts of structures with different time step size and/or different time integration schemes, see e.g. Hughes [8], Hulbert/Hughes [12], it is favorable from a practical point of view to obtain the total information of the status of a structure at a given time. Thus integration schemes with a uniform time step size $\Delta t$ for the 


$$
t=4.0
$$
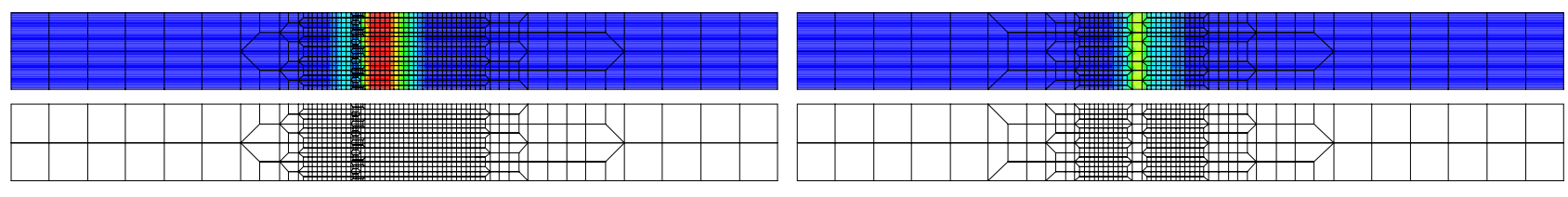

$$
t=9.0
$$
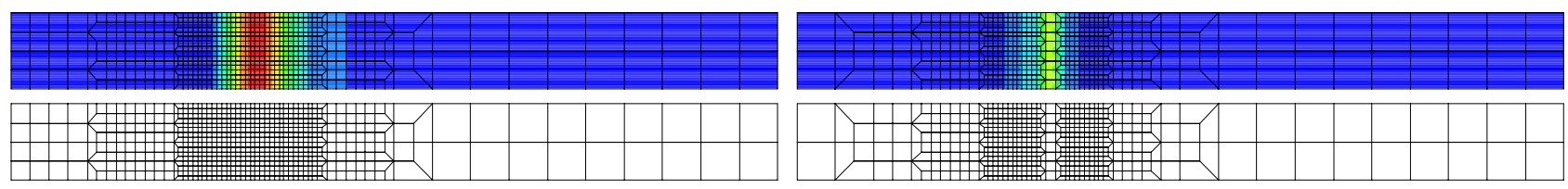

\section{$t=17.2$}

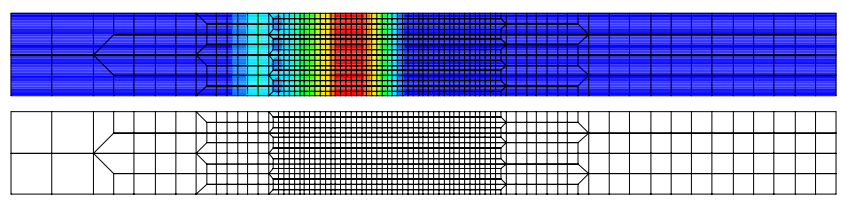

a.) meshes with Newmark-method

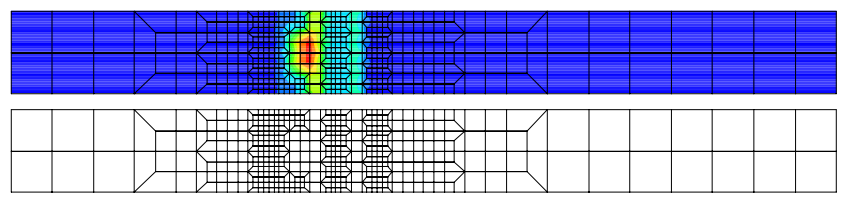

b.) meshes with $D G P 1-P 1$-method

Figure 18: Adaptive meshes at various times created in combination with two different time integration schemes; colored: distribution of the total energy.

complete structure are discussed and our focus is on their properties within a typical interval $I_{n}=\left[t_{n}, t_{n+1}\right]$ with $\Delta t=t_{n+1}-t_{n}$.

The error within an interval can be viewed as local error and its accumulation over the complete process time as global error. The latter is of major interest for the final result and the question is, how to obtain a proper information about this error in order to adjust the time step size. Currently, some global schemes [24], [4] based on duality considerations are developed. However, though they give some hints about the global error, their use for real-size FE problems seems to be limited. Thus, our focus is on the local error estimates and the use of such an estimate for a global estimate and time step adaptation.

The error in displacements and velocities at time $t=t_{n+1}$ is given as:

$$
\mathbf{e}_{n+1}=\mathbf{u}_{e x, n+1}-\mathbf{u}_{h, n+1}, \quad \dot{\mathbf{e}}_{n+1}=\dot{\mathbf{u}}_{e x, n+1}-\dot{\mathbf{u}}_{h, n+1} .
$$

For the $D G P 1-P 1$ method the velocity error is given by the separately interpolated velocity-field $\mathbf{v}$. In order to couple the time integration error with the spatial discretization error the total energy-norm of error can be computed, see Riccius/ Schweizerhof [22]:

$$
\|\mathbf{e}(\Delta t)\|_{t o t a l, \Omega, t_{n+1}}=\left(\int_{B}\left(L \mathbf{e}_{n+1}\right)^{T} \cdot \mathbb{C} L \mathbf{e}_{n+1} d \Omega+\int_{B} \rho<\dot{\mathbf{e}}_{n+1}, \dot{\mathbf{e}}_{n+1}>d \Omega\right)^{1 / 2} .
$$

For the Newmark-scheme a Taylor series expansion can be performed to obtain some information about the local error within a time step, assuming the quantities in the 
previous time steps are computed exactly:

$$
\begin{aligned}
& \mathbf{u}_{e x, n+1} \approx \mathbf{u}_{n}+\Delta t \dot{\mathbf{u}}_{n}+\frac{\Delta t^{2}}{2} \ddot{\mathbf{u}}_{n}+\frac{\Delta t^{3}}{6} \ddot{\mathbf{u}}_{n}+\frac{\Delta t^{4}}{24} \ddot{\mathbf{u}}_{n}+\mathcal{O}\left(\Delta t^{5}\right), \\
& \dot{\mathbf{u}}_{e x, n+1} \approx \dot{\mathbf{u}}_{n}+\Delta t \ddot{\mathbf{u}}_{n}+\frac{\Delta t^{2}}{2} \ddot{\mathbf{u}}_{n}+\frac{\Delta t^{3}}{6} \ddot{\mathbf{u}}_{n}+\mathcal{O}\left(\Delta t^{4}\right) .
\end{aligned}
$$

To eliminate terms of order 3 and 4 in eq. (31) the central difference scheme;

$$
\begin{aligned}
& \ddot{\mathbf{u}}_{n}=\frac{\ddot{\mathbf{u}}_{n+1}-\ddot{\mathbf{u}}_{n-1}}{2 \Delta t}+\mathcal{O}\left(\Delta t^{2}\right), \\
& \ddot{\mathbf{u}}_{n}=\frac{\ddot{\mathbf{u}}_{n+1}-2 \ddot{\mathbf{u}}_{n}+\ddot{\mathbf{u}}_{n-1}}{\Delta t^{2}}+\mathcal{O}\left(\Delta t^{2}\right), \text { is used twice, similar to Li [15]. }
\end{aligned}
$$

Then the displacements and velocities depend only on displacements, velocities and accelerations of the last two time steps plus higher order remainders:

$$
\begin{aligned}
& \mathbf{u}_{e x, n+1} \approx \mathbf{u}_{n}+\Delta t \dot{\mathbf{u}}_{n}+\Delta t^{2}\left(-\frac{1}{24} \ddot{\mathbf{u}}_{n-1}+\frac{5}{12} \ddot{\mathbf{u}}_{n}+\frac{1}{8} \ddot{\mathbf{u}}_{n+1}\right)+\mathcal{O}\left(\Delta t^{5}\right) \\
& \dot{\mathbf{u}}_{e x, n+1} \approx \dot{\mathbf{u}}_{n}+\Delta t\left(-\frac{1}{12} \ddot{\mathbf{u}}_{n-1}+\frac{2}{3} \ddot{\mathbf{u}}_{n}+\frac{5}{12} \ddot{\mathbf{u}}_{n+1}\right)+\mathcal{O}\left(\Delta t^{4}\right) .
\end{aligned}
$$

With the Newmark interpolation for the displacements $\mathbf{u}_{h, n+1}$ and velocities $\dot{\mathbf{u}}_{h, n+1}$, see eq. $(5,6)$ the local error estimate for the Newmark scheme can be given:

$$
\begin{aligned}
\mathbf{e}_{n+1} & =\frac{\Delta t^{2}}{24}\left(\ddot{\mathbf{u}}_{n-1}+(2-24 \beta) \ddot{\mathbf{u}}_{n}+(24 \beta-3) \ddot{\mathbf{u}}_{n+1}\right)+\mathcal{O}\left(\Delta t^{5}\right), \\
\dot{\mathbf{e}}_{n+1} & =\frac{\Delta t}{12}\left(\ddot{\mathbf{u}}_{n-1}+(4-12 \gamma) \ddot{\mathbf{u}}_{n}+(12 \gamma-5) \ddot{\mathbf{u}}_{n+1}\right)+\mathcal{O}\left(\Delta t^{4}\right) .
\end{aligned}
$$

For the $D G P 1-P 1$ scheme a very simple local error indicator can be constructed, see Ruge [23] and Wiberg/Li [16], based on the discontinuity of displacements and velocities:

$$
\mathbf{e}_{n+1}=\mathbf{u}_{n+1}^{+}-\mathbf{u}_{n+1}^{-}, \quad \dot{\mathbf{e}}_{n+1}=\mathbf{v}_{n+1}^{+}-\mathbf{v}_{n+1}^{-} .
$$

However, the latter cannot be used for the global error estimation. As a short remark for the $D G P 2$ method, we have to note that there is no displacement discontinuity in time, because $\mathbf{u}_{n+1}^{+}=\mathbf{u}_{n+1}^{-}$. That is another aspect, why the $D G P 1-P 1$ is superior concerning efficiency when compared to $D G P$ 2. The global error of the $D G$ schemes discussed is certainly also dependent on their properties concerning the high frequency damping, thus global error estimates based on the rather simple local error estimates are difficult and an error estimation function as proposed in [24] seems appropriate for the DG methods.

For the Newmark scheme a rather simple formula for the global time integration error $\|\mathbf{e}(\Delta t)\|_{t o t a l, \Omega, T}$ is based on an extrapolation proposed by Zienkiewicz/Xie [28] and Warburton [25]:

$$
\|\mathbf{e}(\Delta t)\|_{t o t a l, \Omega, T}=\mathcal{N}_{T}\|\mathbf{e}(\Delta t)\|_{t o t a l, \Omega, t_{n+1}}
$$


with $\mathcal{N}_{T}=T / \Delta t_{n}$ as remaining number of time steps until the end of the process.

\section{Time step control}

If a constant time step size is assumed, e.g. $\Delta t_{\text {new }}=c \Delta t_{\text {old }}$, and the order of accuracy of the Newmark scheme is taken into account, we obtain:

$$
\|\mathbf{e}(c \Delta t)\|_{t o t a l, \Omega, T}=c^{2}\|\mathbf{e}(\Delta t)\|_{t o t a l, \Omega, T}
$$

With a required energy norm of the error $\varepsilon_{v, T}$ at the end of the process the constant $c$ can be computed :

$$
c=\left(\frac{\varepsilon_{v, T}}{\|\mathbf{e}(\Delta t)\|_{t o t a l, \Omega, T}}\right)^{1 / 2}=\left(\frac{\varepsilon_{v, T} \Delta t_{\text {old }}}{T\|\mathbf{e}(\Delta t)\|_{\text {total }, \Omega, t_{n+1}}}\right)^{1 / 2} .
$$

For the limitation of the estimated error at the end of the process after eq.(35) an upper limit is given for accuracy and a lower limit for efficiency reasons : $\varepsilon_{\min , T} \leq$ $\|\mathbf{e}(\Delta t)\|_{\text {total }, \Omega, T} \leq \varepsilon_{\max , T}$. The time step size is modified, if any of the tolerances is violated. Then the question remains, how often the time step size has to be adjusted. As the matrices remain constant with constant time step size, then it is advantageous to keep the same time step size, if it is smaller than the required one, as the factorization of the matrices can be avoided then. It is suggested, however, that a time step size adjustment is also performed after each mesh modification.

The same strategy, global error estimation and time step control, was applied as a simple test with the $D G P 1-P 1$ scheme.

As numerical example the clamped plate system, see figure 9 , is taken to show the effects of both error estimators resp. indicators, eq. $(32,33)$ and $(34)$. For both the Newmark and the $D G P 1-P 1$ schemes first a constant and second an adaptively modified time step is used. For comparison also the solution for the velocity $v_{z}$ at the end of the cantilever using modal decomposition of the FE model was computed. In order to get a physically motivated change of the time step size, the modal damping parameters $a$ and $b$ were chosen as: $a=0.4, b=0.0014$. The default resp. starting time step size for all computations was set to $\Delta t=0.1$; the integration interval considered is $t \in[0,5]$.

In figure 19 the evolution of the time step size is presented. It is expected, that the $D G$ P1-P1 method would lead to a larger time step size than the Newmark method due to the higher order accuracy. However, as found also in other numerical examples, the Newmark scheme leads to a larger time step size. The main reason is that the jumps $(\Delta \mathbf{u}, \Delta \mathbf{v})$ give only a restricted information about the error at a discrete time state, whereas the local error estimator for the Newmark scheme describes the error evolution in one time step. The time history plots of the velocity $v_{z}$ at the end of the clamped plate in figure 20a. show the solution with time step adaptation for the Newmark method which approaches the modal solution very well. 
The major improvement of the adaptive solution compared to using a constant time step size is in the beginning of the process, when a small time step is needed. In the time range $t \in[2.5,5.0]$ the solution with constant time step size will also approximate the modal solution, which is caused by the physical damping. For the DG method only very small differences between the solutions with constant and variable time step were obtained, also indicating the higher accuracy properties for the fairly large time steps.

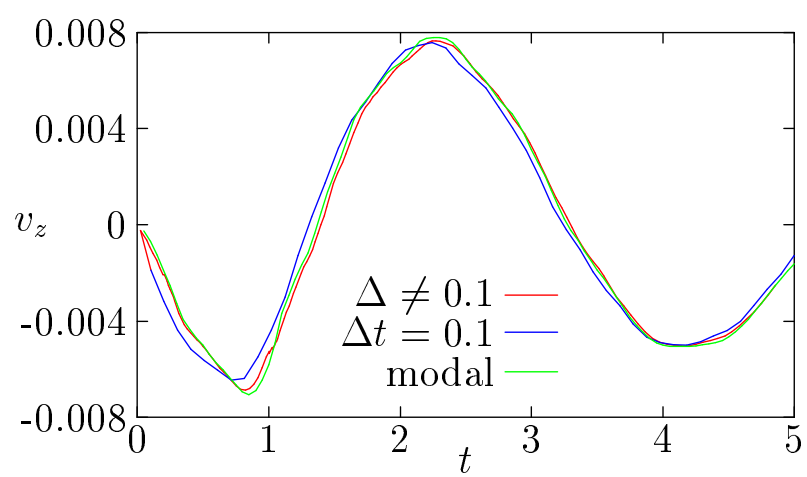

a.) Newmark scheme

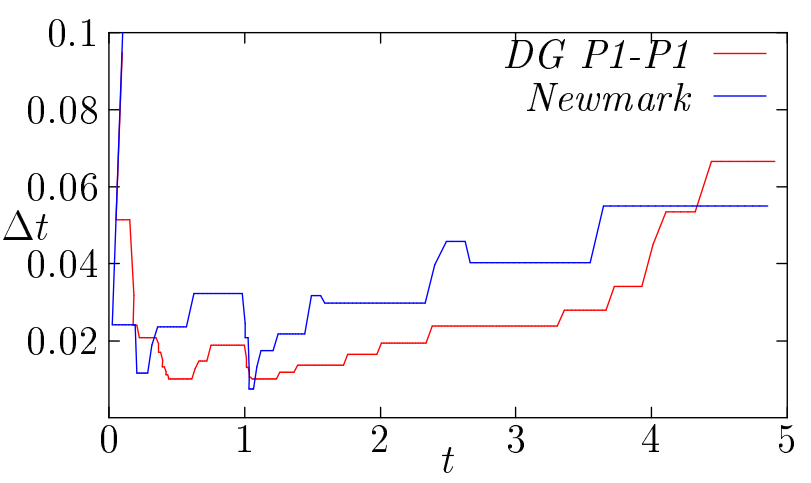

Figure 19: Evolution of the time step size in time for Newmark and $D G P 1-P 1$ methods.

Figure 20: Velocity history at the end of cantilever with and without time step adaptation using DG P1-P1 and Newmark scheme compared to the modal solution.

\section{Conclusions}

The basic properties of two numerical time integration schemes, Discontinuous Galerkin and Newmark method were presented and compared. It was also shown, that the $D G P 1$ P1 scheme has some advantages compared to the $D G$ P2 scheme within the DG family of methods. Then, two representative problems for wave propagation and for stationary vibration were chosen to discuss the properties of the numerical integration schemes. In particular two different proposals for the $D G P 1-P 1$ method by Wiberg/Li and by Ruge were compared concerning efficiency. Further, the artificial dispersion introduced by spatial discretization was investigated on stationary and adaptively modified FE-meshes. It was found that the $D G P 1-P 1$ scheme leads to rather good solutions in adaptively modified meshes for short duration problems, where the numerical damping does not yet affect the global energy too much. Finally, two local error estimators resp. indicators for the time integration error were discussed briefly using a numerical example. 


\section{References}

[1] D. Aharoni and P. Bar-Yoseph. Mixed finite element formulations in the time domain for solution of dynamic problems. Computational Mechanics, 9:359-374, 1992.

[2] Z. Celep and Z.P. Bazant. Spurious reflection of elastic waves due to gradually changing Finite Element size. Int. J. Numer. Methods Eng., 19:631-646, 1983.

[3] J. Chung and G.M. Hulbert. A time integration algorithm for structural dynamics with improved numerical dissipation: The generalized $\alpha$ method. Journal of Applied Mechanics, 60:371-375, 1993.

[4] D. Estep. A posteriori error bounds and global error control for approximations of ordinary differential equations. SIAM Journal of Numer. Analysis, 32(1):1-48, 1995.

[5] K.F. Graff. Wave motion in elastic solids. Ohio State University Press, 1975.

[6] H.M. Hilber and T.J.R. Hughes. Collocation, dissipation and 'overshoot' for time integration schemes in structural dynamics. Earthquake Engrg. Structural Dynam., 6:99-117, 1978.

[7] C. Hoff and P.J. Pahl. Development of an implicit method with numerical dissipation from a generalized single-step algorithm for structural dynamics. Comput. Meth. Appl. Mech. Eng., 67:367-385, 1988.

[8] T.J.R. Hughes. The Finite Element Method - Linear Static and Dynamic Finite Element Analysis. Prentice-Hall International Editions, 1987.

[9] T.J.R. Hughes and G.M. Hulbert. Space-time Finite Element methods for elastodynamics: Formulations and error estimates. Comput. Meth. Appl. Mech. Eng., 62:339-363, 1988 .

[10] G.M. Hulbert. Discontinuity-capturing operators for elastodynamics. Comput. Meth. Appl. Mech. Eng., 96:409-426, 1992.

[11] G.M. Hulbert. Time finite element methods for structural dynamics. Int. J. Numer. Methods Eng., 33:307-331, 1992.

[12] G.M. Hulbert and T.J.R. Hughes. Numerical evaluation and comparison of subcycling algorithms for structural dynamics. Technical Report, Stanford University, pages 165, 1988.

[13] C. Johnson. Discontinous Galerkin finite element methods for second order hyperbolic problems. Comput. Meth. Appl. Mech. Eng., 107:117-129, 1993.

[14] C. Johnson and J. Pitkäranta. An analysis of the discontinous galerkin method for a scalar hyperbolic equation. Mathematics of Computation, 46(173):1-26, 1986. 
[15] X. Li. Adaptive Finite Element Procedures in Structural Mechanics. Doktorarbeit, Chalmers University of Technology, Gothenborg, 1996.

[16] X.D. Li and N.E. Wiberg. Structural dynamic analysis by a time-discontinous Galerkin finite element method. Int. J. Numer. Methods Eng., 39:2131-2152, 1996.

[17] M. Borri, F. Mello and S.N. Atluri. Variational approaches for dynamics and timefinite-elements: numerical studies. Computational Mechanics, 7:49-76, 1990.

[18] R.D. Mindlin. Influence of rotatory inertia and shear in flexural motions of isotropic elastic plates. Journal of Applied Mechanics, 18:31-38, 1951.

[19] N.M. Newmark. A numerical method for structural dynamics. J. Eng. Mech. Div. $A S C E, 85: 67-94,1959$.

[20] L. Nilsson. Impact loading on concrete structures. Phd thesis, Department of Structural Mechanics, Chalmers, Gothenburg, 1979.

[21] J.T. Oden. A general theory of Finite Elements II. Applications. Int. J. Numer. Methods Eng., 1:247-259, 1969.

[22] J. Riccius and K. Schweizerhof. Aspects of hierarchical $h$-adaptive dynamic analyses. In B.H.V. Topping, editor, Third International Conference on Computational Structures Technology. Civil-Comp Press, 1996.

[23] P. Ruge. Hybrid time-finite-elements with time-step-adaption by discontinuity control. Computational Mechanics, 17:392-397, 1996.

[24] F. Cirak, A. Schleupen and E. Ramm. Error estimators for local and global variables using duality principles. Fourth World Congress on Computational Mechanics, Buenos Aires, IACM, 1998.

[25] G.B. Warburton. Formulae for errors for initial displacement and velocity problems using the newmark method. Earthquake Engrg. Structural Dynam., 18:565-573, 1989.

[26] O.C. Zienkiewicz. A new look at the newmark, houbolt and other time stepping formulas. a weighted residual approach. Earthquake Engrg. Structural Dynam., 5:413418, 1977.

[27] O.C. Zienkiewicz and R.L. Taylor. The Finite Element method - vol 1. McGraw-Hill International Editions, 1989.

[28] O.C. Zienkiewicz and Y.M. Xie. A simple local error estimate and an adaptive timestepping procedure for dynamic analysis. Earthquake Engrg. Structural Dynam., 22:871-887, 1991.

[29] O.C. Zienkiewicz and J.Z. Zhu. The superconvergent patch recovery and a posteriori error estimates. part 1: The recovery technique. Int. J. Numer. Methods Eng., 33:1131-1364, 1992. 